\title{
ASYMPTOTIC BEHAVIOR OF CRITICAL INDECOMPOSABLE MULTI-TYPE BRANCHING PROCESSES WITH IMMIGRATION*
}

\author{
TivadAR DANKA ${ }^{1}$ AND Gyula PAP ${ }^{1}$
}

\begin{abstract}
In this paper the asymptotic behavior of a critical multi-type branching process with immigration is described when the offspring mean matrix is irreducible, in other words, when the process is indecomposable. It is proved that sequences of appropriately scaled random step functions formed from periodic subsequences of a critical indecomposable multi-type branching process with immigration converge weakly towards a process supported by a ray determined by the Perron vector of the offspring mean matrix. The types can be partitioned into nonempty mutually disjoint subsets (according to communication of types) such that the coordinate processes belonging to the same subset are multiples of the same squared Bessel process, and the coordinate processes belonging to different subsets are independent.
\end{abstract}

Mathematics Subject Classification. 60J80, 60F17, 60J60.

Received May 13, 2015. Revised December 9, 2015. Accepted March 10, 2016.

\section{INTRODUCTION}

Let $\left(X_{k}\right)_{k \in \mathbb{Z}_{+}}$be a single-type Galton-Watson branching process with immigration given by

$$
X_{k}=\sum_{j=1}^{X_{k-1}} \xi_{k, j}+\varepsilon_{k}, \quad k \in \mathbb{N},
$$

and with initial value $X_{0}=0$, where $X_{k}$ denotes the number of individuals in the $k$ th generation, $\xi_{k, j}$ denotes the number of offsprings produced by the $j$ th individual belonging to the $(k-1)$ th generation, $\varepsilon_{k}$ denotes the number of immigrants in the $k$ th generation, $\left\{\xi_{k, j}, \varepsilon_{k}: k, j \in \mathbb{N}\right\}$ are supposed to be independent, $\left\{\xi_{k, j}: k, j \in \mathbb{N}\right\}$ and $\left\{\varepsilon_{k}: k \in \mathbb{N}\right\}$ are supposed to consist of identically distributed random variables, and $\mathbb{Z}_{+}$and $\mathbb{N}$ denote the set of non-negative integers and positive integers, respectively. Suppose that $\mathbb{E}\left(\xi_{1,1}^{2}\right)<\infty, \mathbb{E}\left(\varepsilon_{1}^{2}\right)<\infty$, and $m_{\xi}:=\mathbb{E}\left(\xi_{1,1}\right)=1$, i.e., the process is critical. Wei and Winnicki [19] proved a functional limit theorem

$$
\mathcal{X}^{(n)} \stackrel{\mathcal{D}}{\longrightarrow} \mathcal{X} \quad \text { as } n \rightarrow \infty,
$$

\footnotetext{
Keywords and phrases. Critical multi-type branching processes with immigration, squared Bessel processes.

* The research of T. Danka and G. Pap was realized in the frames of TÁMOP 4.2.4. A/2-11-1-2012-0001 'National Excellence Program - Elaborating and operating an inland student and researcher personal support system'. The project was subsidized by the European Union and co-financed by the European Social Fund.

1 Bolyai Institute, University of Szeged, Aradi vértanúk tere 1, 6720 Szeged, Hungary. tivadar.danka@math.u-szeged.hu
} 
with $\mathcal{X}_{t}^{(n)}:=n^{-1} X_{\lfloor n t\rfloor}$ for $t \in \mathbb{R}_{+}, n \in \mathbb{N}$, where $\mathbb{R}_{+}$denotes the set of non-negative real numbers, $\lfloor x\rfloor$ denotes the integer part of $x \in \mathbb{R}$, and $\left(\mathcal{X}_{t}\right)_{t \in \mathbb{R}_{+}}$is the pathwise unique strong solution of the stochastic differential equation (SDE)

$$
\mathrm{d} \mathcal{X}_{t}=m_{\varepsilon} \mathrm{d} t+\sqrt{V_{\xi} \mathcal{X}_{t}^{+}} \mathrm{d} \mathcal{W}_{t}, \quad t \in \mathbb{R}_{+},
$$

with initial value $\mathcal{X}_{0}=0$, where $m_{\varepsilon}:=\mathbb{E}\left(\varepsilon_{1}\right), V_{\xi}:=\operatorname{Var}\left(\xi_{1,1}\right),\left(\mathcal{W}_{t}\right)_{t \in \mathbb{R}_{+}}$is a standard Wiener process, and $x^{+}$ denotes the positive part of $x \in \mathbb{R}$.

We will investigate a $p$-type branching process $\left(\boldsymbol{X}_{k}\right)_{k \in \mathbb{Z}_{+}}$with immigration. For simplicity, we suppose that the initial value is $\boldsymbol{X}_{0}=\mathbf{0}$. For each $k \in \mathbb{Z}_{+}$and $i \in\{1, \ldots, p\}$, the number of individuals of type $i$ in the $k$ th generation is denoted by $X_{k, i}$. The non-negative integer-valued random variable $\xi_{k, j, i, \ell}$ denotes the number of type $\ell$ offsprings produced by the $j$ th individual who is of type $i$ belonging to the $(k-1)$ th generation. The number of type $i$ immigrants in the $k$ th generation will be denoted by $\varepsilon_{k, i}$. Consider the random vectors

$$
\boldsymbol{X}_{k}:=\left[\begin{array}{c}
X_{k, 1} \\
\vdots \\
X_{k, p}
\end{array}\right], \quad \boldsymbol{\xi}_{k, j, i}:=\left[\begin{array}{c}
\xi_{k, j, i, 1} \\
\vdots \\
\xi_{k, j, i, p}
\end{array}\right], \quad \boldsymbol{\varepsilon}_{k}:=\left[\begin{array}{c}
\varepsilon_{k, 1} \\
\vdots \\
\varepsilon_{k, p}
\end{array}\right] .
$$

Then we have

$$
\boldsymbol{X}_{k}=\sum_{i=1}^{p} \sum_{j=1}^{X_{k-1, i}} \boldsymbol{\xi}_{k, j, i}+\boldsymbol{\varepsilon}_{k}, \quad k \in \mathbb{N} .
$$

Here $\left\{\boldsymbol{\xi}_{k, j, i}, \boldsymbol{\varepsilon}_{k}: k, j \in \mathbb{N}, i \in\{1, \ldots, p\}\right\}$ are supposed to be independent. Moreover, $\left\{\boldsymbol{\xi}_{k, j, i}: k, j \in \mathbb{N}\right\}$ for each $i \in\{1, \ldots, p\}$, and $\left\{\varepsilon_{k}: k \in \mathbb{N}\right\}$ are supposed to consist of identically distributed vectors. Suppose $\mathbb{E}\left(\left\|\boldsymbol{\xi}_{1,1, i}\right\|^{2}\right)<\infty$ for all $i \in\{1, \ldots, p\}$ and $\mathbb{E}\left(\left\|\varepsilon_{1}\right\|^{2}\right)<\infty$. Introduce the notations

$$
\begin{aligned}
& \boldsymbol{m}_{\boldsymbol{\xi}_{i}}:=\mathbb{E}\left(\boldsymbol{\xi}_{1,1, i}\right) \in \mathbb{R}_{+}^{p}, \quad \boldsymbol{m}_{\boldsymbol{\xi}}:=\left[\boldsymbol{m}_{\boldsymbol{\xi}_{1}} \cdots \boldsymbol{m}_{\boldsymbol{\xi}_{p}}\right] \in \mathbb{R}_{+}^{p \times p}, \quad \boldsymbol{m}_{\boldsymbol{\varepsilon}}:=\mathbb{E}\left(\boldsymbol{\varepsilon}_{1}\right) \in \mathbb{R}_{+}^{p}, \\
& \boldsymbol{V}_{\boldsymbol{\xi}_{i}}:=\operatorname{Var}\left(\boldsymbol{\xi}_{1,1, i}\right) \in \mathbb{R}^{p \times p}, \quad \boldsymbol{V}_{\boldsymbol{\varepsilon}}:=\operatorname{Var}\left(\varepsilon_{1}\right) \in \mathbb{R}^{p \times p} .
\end{aligned}
$$

Note that many authors define the offspring mean matrix as $\boldsymbol{m}_{\boldsymbol{\xi}}^{\top}$. For $k \in \mathbb{Z}_{+}$, let $\mathcal{F}_{k}:=\sigma\left(\boldsymbol{X}_{0}, \boldsymbol{X}_{1}, \ldots, \boldsymbol{X}_{k}\right)$. By (1.2),

$$
\mathbb{E}\left(\boldsymbol{X}_{k} \mid \mathcal{F}_{k-1}\right)=\sum_{i=1}^{p} X_{k-1, i} \boldsymbol{m}_{\xi_{i}}+\boldsymbol{m}_{\boldsymbol{\varepsilon}}=\boldsymbol{m}_{\boldsymbol{\xi}} \boldsymbol{X}_{k-1}+\boldsymbol{m}_{\boldsymbol{\varepsilon}} .
$$

Consequently, $\mathbb{E}\left(\boldsymbol{X}_{k}\right)=\boldsymbol{m}_{\boldsymbol{\xi}} \mathbb{E}\left(\boldsymbol{X}_{k-1}\right)+\boldsymbol{m}_{\varepsilon}$, which implies

$$
\mathbb{E}\left(\boldsymbol{X}_{k}\right)=\sum_{j=0}^{k-1} \boldsymbol{m}_{\xi}^{j} \boldsymbol{m}_{\varepsilon}, \quad k \in \mathbb{N} .
$$

Hence the offspring mean matrix $\boldsymbol{m}_{\boldsymbol{\xi}}$ plays a crucial role in the asymptotic behavior of the sequence $\left(\boldsymbol{X}_{k}\right)_{k \in \mathbb{Z}_{+}}$.

A multi-type branching process $\left(\boldsymbol{X}_{k}\right)_{k \in \mathbb{Z}_{+}}$is referred to respectively as subcritical, critical or supercritical if $\varrho\left(\boldsymbol{m}_{\boldsymbol{\xi}}\right)<1, \varrho\left(\boldsymbol{m}_{\boldsymbol{\xi}}\right)=1$ or $\varrho\left(\boldsymbol{m}_{\boldsymbol{\xi}}\right)>1$, where $\varrho\left(\boldsymbol{m}_{\boldsymbol{\xi}}\right)$ denotes the spectral radius of the offspring mean matrix $\boldsymbol{m}_{\boldsymbol{\xi}}$ (see, e.g., Athreya and Ney [1] or Quine [17]). The process $\left(\boldsymbol{X}_{k}\right)_{k \in \mathbb{Z}_{+}}$is called indecomposable if the matrix $\boldsymbol{m}_{\boldsymbol{\xi}}$ is irreducible. Note that the matrix $\boldsymbol{m}_{\boldsymbol{\xi}}$ is irreducible if and only if for all $i, j \in\{1, \ldots, p\}$ there exists $n_{i, j} \in \mathbb{N}$ such that the matrix entry $\left(\boldsymbol{m}_{\xi}^{n_{i, j}}\right)_{i, j}$ is positive. In other words, a process is indecomposable if and only if each type of individual may have progeny of any other type. An indecomposable process $\left(\boldsymbol{X}_{k}\right)_{k \in \mathbb{Z}_{+}}$is called positively regular if the matrix $\boldsymbol{m}_{\boldsymbol{\xi}}$ is primitive. Note that the matrix $\boldsymbol{m}_{\boldsymbol{\xi}}$ is primitive if and only if there exists $n \in \mathbb{N}$ such that the matrix entry $\left(\boldsymbol{m}_{\xi}^{n}\right)_{i, j}$ is positive. Note that a critical single-type branching process is positively regular. 
Joffe and Métivier ([11], Thm. 4.3.1) studied positively regular critical multi-type branching processes. They determined the limiting behavior of the martingale part $\mathcal{M}^{(n)}, n \in \mathbb{N}$, given by $\mathcal{M}_{t}^{(n)}:=n^{-1} \sum_{k=1}^{\lfloor n t\rfloor} \boldsymbol{M}_{k}$, $t \in \mathbb{R}_{+}$, with $\boldsymbol{M}_{k}:=\boldsymbol{X}_{k}-\mathbb{E}\left(\boldsymbol{X}_{k} \mid \mathcal{F}_{k-1}\right), k \in \mathbb{N}$ (which is a special case of Thm. 3.4).

The result (1.1) has been generalized by Ispány and Pap [8] for positively regular critical $p$-type branching processes with immigration. They proved that

$$
\mathcal{X}^{(n)} \stackrel{\mathcal{D}}{\longrightarrow} \mathcal{X} \boldsymbol{u} \quad \text { as } n \rightarrow \infty,
$$

where $\mathcal{X}_{t}^{(n)}:=n^{-1} \boldsymbol{X}_{\lfloor n t\rfloor}$ for $t \in \mathbb{R}_{+}, n \in \mathbb{N}$, the process $\left(\mathcal{X}_{t}\right)_{t \in \mathbb{R}_{+}}$is the unique strong solution of the SDE

$$
\mathrm{d} \mathcal{X}_{t}=\boldsymbol{v}^{\top} \boldsymbol{m}_{\varepsilon} \mathrm{d} t+\sqrt{\boldsymbol{v}^{\top}\left(\boldsymbol{u} \odot \boldsymbol{V}_{\boldsymbol{\xi}}\right) \boldsymbol{v} \mathcal{X}_{t}^{+}} \mathrm{d} \mathcal{W}_{t}, \quad t \in \mathbb{R}_{+},
$$

with initial value $\mathcal{X}_{0}=0$, where $\boldsymbol{u}$ and $\boldsymbol{v}$ denotes the right and left Perron vectors of $\boldsymbol{m}_{\boldsymbol{\xi}}$, and $\boldsymbol{u} \odot \boldsymbol{V}_{\boldsymbol{\xi}}:=$ $\sum_{i=1}^{p} u_{i} \boldsymbol{V}_{\boldsymbol{\xi}_{i}}$, where $\boldsymbol{u}=\left(u_{i}\right)_{i \in\{1, \ldots, p\}}$.

The aim of the present paper is to obtain a generalization of this result for indecomposable critical multi-type branching processes with immigration. Then the types $\{1, \ldots, p\}$ can be partitioned according to communication of types, namely, into $r$ nonempty mutually disjoint subsets $D_{1}, \ldots, D_{r}$ such that an individual of type $j$ may not have offspring of type $i$ unless there exists $\ell \in\{1, \ldots, r\}$ with $i \in D_{\ell-1}$ and $j \in D_{\ell}$, where subscripts are considered modulo $r$. This partitioning is unique up to cyclic permutation of the subsets. The number $r$ is called the index of cyclicity (in other words, the index of imprimivity) of the matrix $\boldsymbol{m}_{\boldsymbol{\xi}}$. Note that $r=1$ if and only if the matrix $\boldsymbol{m}_{\boldsymbol{\xi}}$ is primitive, i.e., the process is positively regular. We succeeded to determine the joint asymptotic behavior of sequences $\left((n r)^{-1} \boldsymbol{X}_{r\lfloor n t\rfloor+i-1}\right)_{t \in \mathbb{R}_{+}}, n \in \mathbb{N}, i \in\{1, \ldots, r\}$, of random step functions as $n \rightarrow \infty$, see Theorem 3.1. It turns out that the limiting diffusion process has the form $\left(\boldsymbol{m}_{\xi}^{r-i+1} \mathcal{Y}_{t}\right)_{t \in \mathbb{R}_{+}}, i \in\{1, \ldots, r\}$, where the distribution of the process $\left(\boldsymbol{m}_{\xi}^{j} \mathcal{Y}_{t}\right)_{t \in \mathbb{R}_{+}}$is the same for all $j \in \mathbb{N}$. Moreover, the process $\left(\mathcal{Y}_{t}\right)_{t \in \mathbb{R}_{+}}$is 1-dimensional in the sense that for all $t \in \mathbb{R}_{+}$, the distribution of $\mathcal{Y}_{t}$ is concentrated on the ray $\mathbb{R}_{+} \cdot \boldsymbol{u}$, where $\boldsymbol{u}$ is the Perron eigenvector of the offspring mean matrix $\boldsymbol{m}_{\boldsymbol{\xi}}$. In fact, partitioning the coordinates of the limit process $\mathcal{Y}_{t}=\left(\mathcal{Y}_{t, 1}, \ldots, \mathcal{Y}_{t, r}\right)$ and of the Perron eigenvector $\boldsymbol{u}=\left(\boldsymbol{u}_{1}, \ldots, \boldsymbol{u}_{r}\right)$ according to communication of types, we have $\mathcal{Y}_{t, i}=\mathcal{Z}_{t, i} \boldsymbol{u}_{i}, t \in \mathbb{R}_{+}, i \in\{1, \ldots, r\}$, where $\left(\mathcal{Z}_{t, i}\right)_{t \in \mathbb{R}_{+}}, i \in\{1, \ldots, r\}$, are independent squared Bessel processes. It is interesting to note that Kesten and Stigum [14] considered a supercritical indecomposable multi-type branching processes without immigration, and they proved that there exists a random variable $w$ such that $\varrho\left(\boldsymbol{m}_{\xi}\right)^{-(r n+i-1)} \boldsymbol{X}_{r n+i-1} \rightarrow w \boldsymbol{u}_{i}$ almost surely as $n \rightarrow \infty$ for each $i \in\{1, \ldots, r\}$.

The results of the present paper will be very useful for analysing the asymptotic behavior of the conditional least squares estimators of parameters of a critical multi-type branching process with immigration when the process is indecomposable but not positively regular. The positively regular case has been studied in Ispány et al. [9] and in Körmendi and Pap [15].

\section{INDECOMPOSABLE MULTI-TYPE BRANCHING PROCESSES WITH IMMIGRATION}

Let $\mathbb{R}_{++}$denote the set of positive real numbers. The $d$-dimensional unit matrix will be denoted by $\boldsymbol{I}_{d}$. Every random variable will be defined on the fixed probability space $(\Omega, \mathcal{A}, \mathbb{P})$.

In what follows we recall some known facts about irreducible nonnegative matrices. The matrix $\boldsymbol{m}_{\boldsymbol{\xi}}$ is reducible if there exist a permutation matrix $\boldsymbol{P} \in \mathbb{R}^{p \times p}$ and $q \in\{1, \ldots, p-1\}$ such that

$$
\boldsymbol{P}^{\top} \boldsymbol{m}_{\boldsymbol{\xi}} \boldsymbol{P}=\left[\begin{array}{cc}
\boldsymbol{B} & C \\
\mathbf{0} & \boldsymbol{D}
\end{array}\right]
$$

where $\boldsymbol{B} \in \mathbb{R}^{q \times q}, \boldsymbol{D} \in \mathbb{R}^{(p-q) \times(p-q)}, \boldsymbol{C} \in \mathbb{R}^{q \times(p-q)}$ and $\mathbf{0} \in \mathbb{R}^{(p-q) \times q}$ is a null matrix. The matrix $\boldsymbol{m}_{\boldsymbol{\xi}}$ is irreducible if it is not reducible (see, e.g., [6], Defs. 6.2.21 and 6.2.22). If the matrix $\boldsymbol{m}_{\boldsymbol{\xi}}$ is irreducible then, by the Frobenius-Perron theorem the following assertions hold (see, e.g., [2], Thm. 1.8.3, [2], Thm. 2.20, [4], Thm. 8.2.4, [16], Thm. 3.1, or [14]):

- $\varrho\left(\boldsymbol{m}_{\boldsymbol{\xi}}\right) \in \mathbb{R}_{++}, \varrho\left(\boldsymbol{m}_{\boldsymbol{\xi}}\right)$ is an eigenvalue of $\boldsymbol{m}_{\boldsymbol{\xi}}$, and the algebraic and geometric multiplicities of $\varrho\left(\boldsymbol{m}_{\boldsymbol{\xi}}\right)$ equal 1 . 
- Corresponding to the eigenvalue $\varrho\left(\boldsymbol{m}_{\boldsymbol{\xi}}\right)$ there exists a unique (right) eigenvector $\boldsymbol{u} \in \mathbb{R}_{++}^{p}$, called the Perron vector of $\boldsymbol{m}_{\boldsymbol{\xi}}$, such that the sum of the coordinates of $\boldsymbol{u}$ is 1 , and there exists a unique left eigenvector $\boldsymbol{v} \in \mathbb{R}_{++}^{p}$, such that $\boldsymbol{u}^{\top} \boldsymbol{v}=1$.

- If $\boldsymbol{m}_{\boldsymbol{\xi}}$ has exactly $r$ eigenvalues of maximum modulus $\varrho\left(\boldsymbol{m}_{\boldsymbol{\xi}}\right)$ then the coordinates $\{1, \ldots, p\}$ can be partitioned into $r$ nonempty mutually disjoint subsets $D_{1}, \ldots, D_{r}$ such that $m_{i, j}=0$ unless there exists $\ell \in\{1, \ldots, r\}$ with $i \in D_{\ell-1}$ and $j \in D_{\ell}$, where subscripts are considered modulo $r$. This partitioning is unique up to cyclic permutation of the subsets. We may assume that the types are enumerated according to these subsets, and hence

$$
\boldsymbol{m}_{\boldsymbol{\xi}}=\left[\begin{array}{cccccc}
\mathbf{0} & \boldsymbol{m}_{1,2} & \mathbf{0} & \cdots & \mathbf{0} & \mathbf{0} \\
\mathbf{0} & \mathbf{0} & \boldsymbol{m}_{2,3} & \cdots & \mathbf{0} & \mathbf{0} \\
\mathbf{0} & \mathbf{0} & \mathbf{0} & \cdots & \mathbf{0} & \mathbf{0} \\
\vdots & \vdots & \vdots & \ddots & \vdots & \vdots \\
\mathbf{0} & \mathbf{0} & \mathbf{0} & \cdots & \mathbf{0} & \boldsymbol{m}_{r-1, r} \\
\boldsymbol{m}_{r, 1} & \mathbf{0} & \mathbf{0} & \cdots & \mathbf{0} & \mathbf{0}
\end{array}\right]
$$

where the $r$ main diagonal zero blocks are square, $\boldsymbol{m}_{1,2} \in \mathbb{R}^{\left|D_{1}\right| \times\left|D_{2}\right|}, \boldsymbol{m}_{2,3} \in \mathbb{R}^{\left|D_{2}\right| \times\left|D_{3}\right|}, \ldots, \boldsymbol{m}_{r, 1} \in$ $\mathbb{R}^{\left|D_{r}\right| \times\left|D_{1}\right|}$ where $|H|$ denotes the number of elements of a set $H$, and $\boldsymbol{m}_{1,2} \neq \mathbf{0}, \boldsymbol{m}_{2,3} \neq \mathbf{0}, \ldots, \boldsymbol{m}_{r, 1} \neq \mathbf{0}$. Then for each $k \in\{1, \ldots, r-1\}$, we have

$$
\boldsymbol{m}_{\boldsymbol{\xi}}^{k}=\left[\begin{array}{ccccccc}
\mathbf{0} & \cdots & \mathbf{0} & \widetilde{\boldsymbol{m}}_{1, k+1} & \mathbf{0} & \cdots & \mathbf{0} \\
\mathbf{0} & \cdots & \mathbf{0} & \mathbf{0} & \widetilde{\boldsymbol{m}}_{2, k+2} & \cdots & \mathbf{0} \\
\vdots & & \vdots & \vdots & \vdots & \ddots & \vdots \\
\mathbf{0} & \cdots & \mathbf{0} & \mathbf{0} & \mathbf{0} & \cdots & \widetilde{\boldsymbol{m}}_{r-k, r} \\
\widetilde{\boldsymbol{m}}_{r-k+1, r+1} & \cdots & \mathbf{0} & \mathbf{0} & \mathbf{0} & \cdots & \mathbf{0} \\
\vdots & \ddots & \vdots & \vdots & \vdots & & \vdots \\
\mathbf{0} & \cdots & \widetilde{\boldsymbol{m}}_{r, r+k} & \mathbf{0} & \mathbf{0} & \cdots & \mathbf{0}
\end{array}\right]
$$

with

$$
\widetilde{\boldsymbol{m}}_{i, j}:=\boldsymbol{m}_{i, i+1} \boldsymbol{m}_{i+1, i+2} \cdots \boldsymbol{m}_{j-1, j} \in \mathbb{R}^{\left|D_{i}\right| \times\left|D_{j}\right|}
$$

for $i, j \in \mathbb{N}$ with $i<j$, where subscripts on the right hand side are considered modulo $r$. We will also use the notational convention $\widetilde{\boldsymbol{m}}_{i, i}:=\boldsymbol{I}_{\left|D_{i}\right|}$. Moreover,

$$
\boldsymbol{m}_{\boldsymbol{\xi}}^{r}=\left[\begin{array}{cccc}
\widetilde{\boldsymbol{m}}_{1, r+1} & \mathbf{0} & \cdots & \mathbf{0} \\
\mathbf{0} & \widetilde{\boldsymbol{m}}_{2, r+2} & \cdots & \mathbf{0} \\
\vdots & \vdots & \ddots & \vdots \\
\mathbf{0} & \mathbf{0} & \cdots & \widetilde{\boldsymbol{m}}_{r, 2 r}
\end{array}\right]=: \widetilde{\boldsymbol{m}}_{1, r+1} \oplus \widetilde{\boldsymbol{m}}_{2, r+2} \oplus \cdots \oplus \widetilde{\boldsymbol{m}}_{r, 2 r}
$$

The matrices $\widetilde{\boldsymbol{m}}_{1, r+1} \in \mathbb{R}^{\left|D_{1}\right| \times\left|D_{1}\right|}, \widetilde{\boldsymbol{m}}_{2, r+2} \in \mathbb{R}^{\left|D_{2}\right| \times\left|D_{2}\right|}, \ldots, \widetilde{\boldsymbol{m}}_{r, 2 r} \in \mathbb{R}^{\left|D_{r}\right| \times\left|D_{r}\right|}$ are primitive (that is, irreducible and there exists $n_{i} \in \mathbb{N}$ such that $\left.\widetilde{\boldsymbol{m}}_{i, r+i}^{n_{i}} \in \mathbb{R}_{++}^{\left|D_{i}\right| \times\left|D_{i}\right|}\right)$ with $\varrho\left(\widetilde{\boldsymbol{m}}_{i, r+i}\right)=\left[\varrho\left(\boldsymbol{m}_{\boldsymbol{\xi}}\right)\right]^{r}, i \in\{1, \ldots, r\}$. (see, e.g., [16], Thm. 4.3).

- If

$$
\boldsymbol{u}=\left[\begin{array}{c}
\boldsymbol{u}_{1} \\
\vdots \\
\boldsymbol{u}_{r}
\end{array}\right], \quad \boldsymbol{v}=\left[\begin{array}{c}
\boldsymbol{v}_{1} \\
\vdots \\
\boldsymbol{v}_{r}
\end{array}\right]
$$

denotes the partitioning of $\boldsymbol{u}$ and $\boldsymbol{v}$ with respect to the partitioning $D_{1}, \ldots, D_{r}$ of the coordinates $\{1, \ldots, p\}$ then, for each $i \in\{1, \ldots, r\}$, we have $\boldsymbol{u}_{i}^{\top} \boldsymbol{v}_{i}=r^{-1}$, the vectors $\widetilde{\boldsymbol{u}}_{i}:=r \boldsymbol{u}_{i}$ and $\widetilde{\boldsymbol{v}}_{i}:=\boldsymbol{v}_{i}$ are right and left eigenvectors of $\widetilde{\boldsymbol{m}}_{i, r+i}$ with $\widetilde{\boldsymbol{u}}_{i}^{\top} \widetilde{\boldsymbol{v}}_{i}=1$, and

$$
\left[\varrho\left(\widetilde{\boldsymbol{m}}_{i, r+i}\right)\right]^{-n} \widetilde{\boldsymbol{m}}_{i, r+i}^{n} \rightarrow \widetilde{\boldsymbol{u}}_{i} \widetilde{\boldsymbol{v}}_{i}^{\top}=r \boldsymbol{u}_{i} \boldsymbol{v}_{i}^{\top}=: \boldsymbol{\Pi}_{i} \in \mathbb{R}_{++}^{\left|D_{i}\right| \times\left|D_{i}\right|} \quad \text { as } n \rightarrow \infty .
$$


Consequently,

$$
\left[\varrho\left(\boldsymbol{m}_{\boldsymbol{\xi}}\right)\right]^{-n r} \boldsymbol{m}_{\xi}^{n r} \rightarrow \boldsymbol{\Pi} \in \mathbb{R}_{+}^{p \times p} \quad \text { as } n \rightarrow \infty,
$$

where

$$
\boldsymbol{\Pi}:=\boldsymbol{\Pi}_{1} \oplus \boldsymbol{\Pi}_{2} \oplus \cdots \oplus \boldsymbol{\Pi}_{r}=\left[\begin{array}{cccc}
\boldsymbol{\Pi}_{1} & \mathbf{0} & \cdots & \mathbf{0} \\
\mathbf{0} & \boldsymbol{\Pi}_{2} & \cdots & \mathbf{0} \\
\vdots & \vdots & \ddots & \vdots \\
\mathbf{0} & \mathbf{0} & \cdots & \boldsymbol{\Pi}_{r}
\end{array}\right] .
$$

The vectors $\boldsymbol{u}$ and $\boldsymbol{v}$ are right and left eigenvectors of $\boldsymbol{\Pi}$ corresponding to the eigenvalue $\left[\varrho\left(\boldsymbol{m}_{\boldsymbol{\xi}}\right)\right]^{r}$.

- Moreover, there exist $c, \kappa \in \mathbb{R}_{++}$with $\kappa<1$ such that

$$
\left\|\left[\varrho\left(\boldsymbol{m}_{\xi}\right)\right]^{-n r} \boldsymbol{m}_{\xi}^{n r}-\boldsymbol{\Pi}\right\| \leqslant c \kappa^{n} \quad \text { for all } n \in \mathbb{N},
$$

where $\|\boldsymbol{A}\|$ denotes the operator norm of a matrix $\boldsymbol{A} \in \mathbb{R}^{p \times p}$ defined by $\|\boldsymbol{A}\|:=\sup _{\|\boldsymbol{x}\|=1}\|\boldsymbol{A} \boldsymbol{x}\|$.

If $\boldsymbol{m}_{\boldsymbol{\xi}}$ has the form (2.1), then the offsprings have the property $\xi_{1,1, i, j}=0$ almost surely unless there exists $\ell \in\{1, \ldots, r\}$ with $i \in D_{\ell-1}$ and $j \in D_{\ell}$, where $D_{0}:=D_{r}$. Consequently, the offspring variance matrices $\boldsymbol{V}_{\boldsymbol{\xi}_{j}}$, $j \in\{1, \ldots, p\}$, have the form

$$
\boldsymbol{V}_{\boldsymbol{\xi}_{j}}= \begin{cases}\mathbf{0} \oplus \mathbf{0} \oplus \cdots \oplus \mathbf{0} \oplus \boldsymbol{V}_{1, j} & \text { if } j \in D_{1}, \\ \boldsymbol{V}_{2, j} \oplus \mathbf{0} \oplus \cdots \oplus \mathbf{0} \oplus \mathbf{0} & \text { if } j \in D_{2}, \\ \vdots & \\ \mathbf{0} \oplus \mathbf{0} \oplus \cdots \oplus \boldsymbol{V}_{r, j} \oplus \mathbf{0} & \text { if } j \in D_{r},\end{cases}
$$

where $\boldsymbol{V}_{\ell, j} \in \mathbb{R}^{\left|D_{\ell-1}\right| \times\left|D_{\ell-1}\right|}$ denotes the variance matrix of the random vector $\left(\xi_{1,1, j, i}\right)_{i \in D_{\ell-1}}$ for $\ell \in\{1, \ldots, r\}$, $j \in D_{\ell}$. For a vector $\boldsymbol{\alpha}_{\ell}=\left(\alpha_{\ell, j}\right)_{j \in D_{\ell}} \in \mathbb{R}_{+}^{\left|D_{\ell}\right|}$ with $\ell \in\{1, \ldots, r\}$, we will use notation $\boldsymbol{\alpha}_{\ell} \odot \boldsymbol{V}_{\ell}:=$ $\sum_{j \in D_{\ell}} \alpha_{\ell, j} \boldsymbol{V}_{\ell, j} \in \mathbb{R}^{\left|D_{\ell-1}\right| \times\left|D_{\ell-1}\right|}$, which is a positive semi-definite matrix, a mixture of the variance matrices $\boldsymbol{V}_{\ell, j}, j \in D_{\ell}$. For a vector $\boldsymbol{\alpha}=\left(\alpha_{i}\right)_{i \in\{1, \ldots, p\}} \in \mathbb{R}_{+}^{p}$, we will also use the notation $\boldsymbol{\alpha} \odot \boldsymbol{V}_{\boldsymbol{\xi}}:=\sum_{i=1}^{p} \alpha_{i} \boldsymbol{V}_{\boldsymbol{\xi}_{i}} \in \mathbb{R}^{p \times p}$, which is a positive semi-definite matrix, a mixture of the variance matrices $\boldsymbol{V}_{\xi_{1}}, \ldots, \boldsymbol{V}_{\xi_{p}}$.

\section{Convergence Results}

A function $f: \mathbb{R}_{+} \rightarrow \mathbb{R}^{d}$ is called càdlàg if it is right continuous with left limits. Let $\mathrm{D}\left(\mathbb{R}_{+}, \mathbb{R}^{d}\right)$ and $\mathrm{C}\left(\mathbb{R}_{+}, \mathbb{R}^{d}\right)$ denote the space of all $\mathbb{R}^{d}$-valued càdlàg and continuous functions on $\mathbb{R}_{+}$, respectively. Let $\mathcal{D}_{\infty}\left(\mathbb{R}_{+}, \mathbb{R}^{d}\right)$ denote the Borel $\sigma$-algebra in $\mathrm{D}\left(\mathbb{R}_{+}, \mathbb{R}^{d}\right)$ for the metric defined in ([10], Chap. VI, Eq. (1.26)) (with this metric $\mathrm{D}\left(\mathbb{R}_{+}, \mathbb{R}^{d}\right)$ is a complete and separable metric space). For $\mathbb{R}^{d}$-valued stochastic processes $\left(\mathcal{Y}_{t}\right)_{t \in \mathbb{R}_{+}}$ and $\left(\mathcal{Y}_{t}^{(n)}\right)_{t \in \mathbb{R}_{+}}, n \in \mathbb{N}$, with càdlàg paths we write $\mathcal{Y}^{(n)} \stackrel{\mathcal{D}}{\longrightarrow} \mathcal{Y}$ if the distribution of $\mathcal{Y}^{(n)}$ on the space $\left(\mathrm{D}\left(\mathbb{R}_{+}, \mathbb{R}^{d}\right), \mathcal{D}_{\infty}\left(\mathbb{R}_{+}, \mathbb{R}^{d}\right)\right)$ converges weakly to the distribution of $\mathcal{Y}$ on the space $\left(\mathrm{D}\left(\mathbb{R}_{+}, \mathbb{R}^{d}\right), \mathcal{D}_{\infty}\left(\mathbb{R}_{+}, \mathbb{R}^{d}\right)\right)$ as $n \rightarrow \infty$.

Theorem 3.1. Let $\left(\boldsymbol{X}_{k}\right)_{k \in \mathbb{Z}_{+}}$be a indecomposable critical p-type branching process with immigration. Suppose $\boldsymbol{X}_{0}=\mathbf{0}, \mathbb{E}\left(\left\|\boldsymbol{\xi}_{1,1, i}\right\|^{2}\right)<\infty$ for all $i \in\{1, \ldots, p\}$ and $\mathbb{E}\left(\left\|\varepsilon_{1}\right\|^{2}\right)<\infty$. Suppose that the index of cyclicity of $\boldsymbol{m}_{\boldsymbol{\xi}}$ is $r \in \mathbb{N}$. Suppose that the offspring mean matrix $\boldsymbol{m}_{\boldsymbol{\xi}}$ has the form (2.1). For each $n \in \mathbb{N}$, consider the rp-dimensional random step process

$$
\boldsymbol{\mathcal { X }}_{t}^{(n)}:=\frac{1}{r n}\left[\begin{array}{c}
\boldsymbol{X}_{r\lfloor n t\rfloor} \\
\boldsymbol{X}_{r\lfloor n t\rfloor-1} \\
\vdots \\
\boldsymbol{X}_{r\lfloor n t\rfloor-r+1}
\end{array}\right], \quad t \in \mathbb{R}_{+} .
$$


Then

$$
\boldsymbol{\mathcal { X }}^{(n)} \stackrel{\mathcal{D}}{\longrightarrow} \mathcal{X} \quad \text { as } n \rightarrow \infty,
$$

where

$$
\mathcal{X}_{t}:=\left[\begin{array}{c}
\boldsymbol{m}_{\xi}^{r} \mathcal{Y}_{t} \\
\boldsymbol{m}_{\xi}^{r-1} \mathcal{Y}_{t} \\
\vdots \\
\boldsymbol{m}_{\xi} \mathcal{Y}_{t}
\end{array}\right], \quad t \in \mathbb{R}_{+}
$$

with

$$
\mathcal{Y}_{t}:=\left[\begin{array}{c}
\mathcal{Y}_{t, 1} \\
\mathcal{Y}_{t, 2} \\
\vdots \\
\mathcal{Y}_{t, r}
\end{array}\right], \quad t \in \mathbb{R}_{+},
$$

where, for $i \in\{1, \ldots, r\}$, the $\left|D_{i}\right|$-dimensional process $\left(\mathcal{Y}_{t, i}\right)_{t \in \mathbb{R}_{+}}$is given by

$$
\mathcal{Y}_{t, i}:=\mathcal{Z}_{t, i} \boldsymbol{u}_{i}, \quad t \in \mathbb{R}_{+},
$$

where $\left(\mathcal{Z}_{t, i}\right)_{t \in \mathbb{R}_{+}}$is the unique strong solution of the $S D E$

$$
\mathrm{d} \mathcal{Z}_{t, i}=\boldsymbol{v}_{i}^{\top} \boldsymbol{m}_{\xi, \varepsilon, i} \mathrm{~d} t+\sqrt{\boldsymbol{v}_{i}^{\top} \boldsymbol{V}_{\boldsymbol{\xi}, \boldsymbol{\varepsilon}, i} \boldsymbol{v}_{i} \mathcal{Z}_{t, i}^{+}} \mathrm{d} \mathcal{W}_{t, i}, \quad t \in \mathbb{R}_{+},
$$

with initial value $\mathcal{Z}_{0, i}=0$, where $\left(\mathcal{W}_{t, i}\right)_{t \in \mathbb{R}_{+}}, i \in\{1, \ldots, r\}$, are independent standard Wiener processes and

$$
\boldsymbol{m}_{\boldsymbol{\xi}, \boldsymbol{\varepsilon}, i}:=\frac{1}{r} \sum_{\ell=i}^{i+r-1} \widetilde{\boldsymbol{m}}_{i, \ell} \boldsymbol{m}_{\boldsymbol{\varepsilon}, \ell}, \quad \boldsymbol{V}_{\boldsymbol{\xi}, \boldsymbol{\varepsilon}, i}:=\frac{1}{r} \sum_{\ell=i}^{i+r-1} \widetilde{\boldsymbol{m}}_{i, \ell}\left[\left(\widetilde{\boldsymbol{m}}_{\ell+1, i+r} \boldsymbol{u}_{i}\right) \odot \boldsymbol{V}_{\ell+1}\right] \widetilde{\boldsymbol{m}}_{i, \ell}^{\top}
$$

where

$$
\boldsymbol{m}_{\varepsilon}=\left[\begin{array}{c}
\boldsymbol{m}_{\varepsilon, 1} \\
\boldsymbol{m}_{\varepsilon, 2} \\
\vdots \\
\boldsymbol{m}_{\boldsymbol{\varepsilon}, r}
\end{array}\right]
$$

denotes the partitioning of $\boldsymbol{m}_{\varepsilon}$ with respect to the partitioning $D_{1}, \ldots, D_{r}$ of the types $\{1, \ldots, p\}$. Here the second subscript of $\boldsymbol{m}_{\boldsymbol{\varepsilon}, \ell}$ in the definition of $\boldsymbol{m}_{\boldsymbol{\xi}, \boldsymbol{\varepsilon}, i}$ and the subscript of $\boldsymbol{V}_{\ell+1}$ in the definition of $\boldsymbol{V}_{\boldsymbol{\xi}, \boldsymbol{\varepsilon}, i}$ are considered modulo $r$. Moreover, the p-dimensional coordinate blocks of the rp-dimensional process $\left(\mathcal{X}_{t}\right)_{t \in \mathbb{R}_{+}}$ have the same distribution, i.e., $\left(\boldsymbol{m}_{\xi}^{i} \mathcal{Y}_{t}\right)_{t \in \mathbb{R}_{+}} \stackrel{\mathcal{D}}{=}\left(\mathcal{Y}_{t}\right)_{t \in \mathbb{R}_{+}}$for all $i \in\{1, \ldots, r-1\}$, and they are periodic, i.e., $\left(\boldsymbol{m}_{\xi}^{r} \mathcal{Y}_{t}\right)_{t \in \mathbb{R}_{+}}=\left(\mathcal{Y}_{t}\right)_{t \in \mathbb{R}_{+}}$almost surely.

Remark 3.2. The $\operatorname{SDE}(3.3)$ has a unique strong solution $\left(\mathcal{Z}_{t, i}^{\left(z_{0}\right)}\right)_{t \in \mathbb{R}_{+}}$for all initial values $\mathcal{Z}_{0, i}^{\left(z_{0}\right)}=z_{0} \in \mathbb{R}$. Indeed, the coefficient functions satisfy conditions of part (ii) of Theorem 3.5 in Chapter IX in [18] or the conditions of Proposition 5.2.13 in Karatzas and Shreve [13]. Further, by the comparison theorem (see, e.g., [18], Thm. 3.7, Chap. IX), if the initial value $\mathcal{Z}_{0, i}^{\left(z_{0}\right)}=z_{0}$ is nonnegative, then $\mathcal{Z}_{t, i}^{\left(z_{0}\right)}$ is nonnegative for all $t \in \mathbb{R}_{+}$with probability one. Hence $\mathcal{Z}_{t, i}^{+}$may be replaced by $\mathcal{Z}_{t, i}$ under the square root in (3.3).

Remark 3.3. Note that Theorem 3.1 implies the convergence result of Ispány and Pap [8] for a positively regular critical $p$-type branching process $\left(\boldsymbol{X}_{k}\right)_{k \in \mathbb{Z}_{+}}$with immigration. Indeed, in this case the index of cyclicity is $r=1$, and $\boldsymbol{m}_{\boldsymbol{\xi}, \boldsymbol{\varepsilon}, 1}=\boldsymbol{m}_{\boldsymbol{\varepsilon}, 1}, \boldsymbol{V}_{\boldsymbol{\xi}, \boldsymbol{\varepsilon}, 1}=\boldsymbol{u} \odot \boldsymbol{V}_{\boldsymbol{\xi}}$. In fact, the result of Ispány and Pap [8] has been proven under the higher moment assumptions $\mathbb{E}\left(\left\|\boldsymbol{\xi}_{1,1, i}\right\|^{4}\right)<\infty$ for all $i \in\{1, \ldots, p\}$ and $\mathbb{E}\left(\left\|\varepsilon_{1}\right\|^{4}\right)<\infty$. Moreover, Theorem 3.1 also implies the convergence result of ([3], Thm. 3.1) for a primitive $\operatorname{INAR}(p)$ process. Eventually, Theorem 3.1 yields a convergence result for an arbitrary $\operatorname{INAR}(p)$ process as well. 
In order to prove (3.1), introduce the $r p$-dimensional random vectors

$$
\boldsymbol{M}_{k}:=\left[\begin{array}{c}
\boldsymbol{M}_{k, 1} \\
\boldsymbol{M}_{k, 2} \\
\vdots \\
\boldsymbol{M}_{k, r}
\end{array}\right]:=\left[\begin{array}{c}
\boldsymbol{X}_{r k}-\mathbb{E}\left(\boldsymbol{X}_{r k} \mid \mathcal{F}_{r k-1}\right) \\
\boldsymbol{X}_{r k-1}-\mathbb{E}\left(\boldsymbol{X}_{r k-1} \mid \mathcal{F}_{r k-2}\right) \\
\vdots \\
\boldsymbol{X}_{r k-r+1}-\mathbb{E}\left(\boldsymbol{X}_{r k-r+1} \mid \mathcal{F}_{r k-r}\right)
\end{array}\right]=\left[\begin{array}{c}
\boldsymbol{X}_{r k}-\boldsymbol{m}_{\boldsymbol{\xi}} \boldsymbol{X}_{r k-1}-\boldsymbol{m}_{\boldsymbol{\varepsilon}} \\
\boldsymbol{X}_{r k-1}-\boldsymbol{m}_{\boldsymbol{\xi}} \boldsymbol{X}_{r k-2}-\boldsymbol{m}_{\boldsymbol{\varepsilon}} \\
\vdots \\
\boldsymbol{X}_{r k-r+1}-\boldsymbol{m}_{\boldsymbol{\xi}} \boldsymbol{X}_{r k-r}-\boldsymbol{m}_{\boldsymbol{\varepsilon}}
\end{array}\right]
$$

for $k \in \mathbb{N}$, forming a sequence of martingale differences with respect to the filtration $\left(\mathcal{F}_{r k}\right)_{k \in \mathbb{Z}_{+}}$. Consider the $r p$-dimensional random step processes

$$
\mathcal{M}_{t}^{(n)}:=\left[\begin{array}{c}
\mathcal{M}_{t, 1}^{(n)} \\
\vdots \\
\mathcal{M}_{t, r}^{(n)}
\end{array}\right]:=\frac{1}{r n} \sum_{k=1}^{\lfloor n t\rfloor} \boldsymbol{M}_{k}, \quad t \in \mathbb{R}_{+}, \quad n \in \mathbb{N} .
$$

The following convergence result is an important step in the proof of Theorem 3.1.

Theorem 3.4. Under the assumptions of Theorem 3.1, we have

$$
\mathcal{M}^{(n)} \stackrel{\mathcal{D}}{\longrightarrow} \mathcal{M} \quad \text { as } n \rightarrow \infty,
$$

where

$$
\mathcal{M}_{t}:=\left[\begin{array}{c}
\mathcal{M}_{t, 1} \\
\vdots \\
\mathcal{M}_{t, r}
\end{array}\right] \quad t \in \mathbb{R}_{+}
$$

is the unique strong solution of the SDE

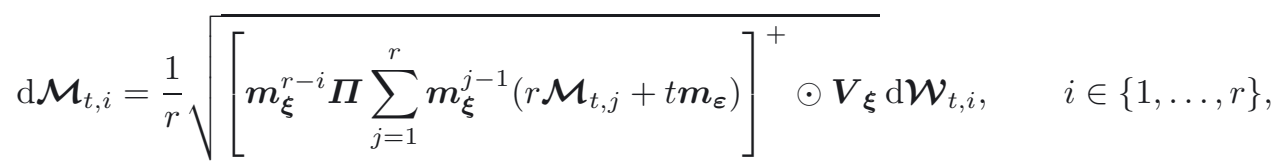

with initial value $\mathcal{M}_{0}=\mathbf{0}$, where $\left(\mathcal{W}_{t, i}\right)_{t \in \mathbb{R}_{+}}, i \in\{1, \ldots, r\}$, are independent $p$-dimensional standard Wiener processes, and for a positive semi-definite matrix $\boldsymbol{A} \in \mathbb{R}^{p \times p}, \sqrt{\boldsymbol{A}}$ denotes its unique symmetric positive semidefinite square root.

In order to handle the $\mathrm{SDE}$ (3.5), consider the $p$-dimensional process

$$
\mathcal{N}_{t}:=\mathcal{M}_{t, 1}+\boldsymbol{m}_{\boldsymbol{\xi}} \mathcal{M}_{t, 2}+\cdots+\boldsymbol{m}_{\boldsymbol{\xi}}^{r-1} \mathcal{M}_{t, r}, \quad t \in \mathbb{R}_{+} .
$$

Theorem 3.5. Under the assumptions of Theorem 3.1, the process $\left(\mathcal{N}_{t}\right)_{t \in \mathbb{R}_{+}}$is the unique strong solution of the $S D E$

$$
\mathrm{d} \mathcal{N}_{t}=\frac{1}{r} \sum_{j=1}^{r} \boldsymbol{m}_{\boldsymbol{\xi}}^{j-1} \sqrt{\left[\boldsymbol{m}_{\boldsymbol{\xi}}^{r-j} \boldsymbol{\Pi}\left(r \mathcal{N}_{t}+t \sum_{\ell=1}^{r} \boldsymbol{m}_{\boldsymbol{\xi}}^{\ell-1} \boldsymbol{m}_{\boldsymbol{\varepsilon}}\right)\right]^{+} \odot \boldsymbol{V}_{\boldsymbol{\xi}}} \mathrm{d} \mathcal{W}_{t, j}, \quad t \in \mathbb{R}_{+},
$$

with initial value $\mathcal{N}_{0}=\mathbf{0}$, and

$$
\mathcal{M}_{t, i}=\frac{1}{r} \int_{0}^{t} \sqrt{\left[\boldsymbol{m}_{\boldsymbol{\xi}}^{r-i} \boldsymbol{\Pi}\left(r \mathcal{N}_{s}+s \sum_{\ell=1}^{r} \boldsymbol{m}_{\boldsymbol{\xi}}^{\ell-1} \boldsymbol{m}_{\varepsilon}\right)\right]^{+} \odot \boldsymbol{V}_{\boldsymbol{\xi}}} \mathrm{d} \mathcal{W}_{s, i}, \quad i \in\{1, \ldots, r\}
$$


If

$$
\mathcal{N}_{t}=\left[\begin{array}{c}
\mathcal{N}_{t, 1} \\
\vdots \\
\mathcal{N}_{t, r}
\end{array}\right], \quad \mathcal{W}_{t, j}=\left[\begin{array}{c}
\mathcal{W}_{t, j, 1} \\
\vdots \\
\mathcal{W}_{t, j, r}
\end{array}\right], \quad j \in\{1, \ldots, r\}, \quad t \in \mathbb{R}_{+}
$$

denote the partitioning of $\mathcal{N}_{t}$ and $\mathcal{W}_{t, j}, j \in\{1, \ldots, r\}$, with respect to the partitioning $D_{1}, \ldots, D_{r}$ of the coordinates $\{1, \ldots, p\}$, then the process $\left(\mathcal{N}_{t}\right)_{t \in \mathbb{R}_{+}}$is the unique strong solution of the $S D E$

$$
\mathrm{d} \mathcal{N}_{t, i}=\sqrt{\left[\boldsymbol{v}_{i}^{\top}\left(\mathcal{N}_{t, i}+\frac{t}{r} \sum_{\ell=i}^{i+r-1} \widetilde{\boldsymbol{m}}_{i, \ell} \boldsymbol{m}_{\boldsymbol{\varepsilon}, \ell}\right)\right]^{+}} \sum_{\ell=i}^{i+r-1} \widetilde{\boldsymbol{m}}_{i, \ell} \sqrt{\left(\widetilde{\boldsymbol{m}}_{\ell+1, i} \boldsymbol{u}_{i}\right) \odot \boldsymbol{V}_{\ell+1}} \mathrm{~d} \mathcal{W}_{t, \ell+i-1, \ell+1},
$$

$i \in\{1, \ldots, r\}$, where the second subscript of $\boldsymbol{m}_{\boldsymbol{\varepsilon}, \ell}$, and the second and third subscripts of $\mathcal{W}_{t, \ell+i-1, \ell+1}$ are considered modulo $r$.

From (3.4) we obtain the recursion $\boldsymbol{X}_{r k-i+1}=\boldsymbol{m}_{\boldsymbol{\xi}} \boldsymbol{X}_{r k-i}+\boldsymbol{M}_{k, i}+\boldsymbol{m}_{\boldsymbol{\varepsilon}}$ for $k \in \mathbb{N}$ and $i \in\{1, \ldots, r\}$, and hence

$$
\boldsymbol{X}_{r-i+1}=\sum_{\ell=i}^{r} \boldsymbol{m}_{\xi}^{\ell-i}\left(M_{1, \ell}+\boldsymbol{m}_{\varepsilon}\right)
$$

for $i \in\{1, \ldots, r\}$, and

$$
\boldsymbol{X}_{r k-i+1}=\boldsymbol{m}_{\boldsymbol{\xi}}^{r} \boldsymbol{X}_{r k-r-i+1}+\sum_{\ell=i}^{r} \boldsymbol{m}_{\boldsymbol{\xi}}^{\ell-i}\left(\boldsymbol{M}_{k, \ell}+\boldsymbol{m}_{\boldsymbol{\varepsilon}}\right)+\sum_{\ell=1}^{i-1} \boldsymbol{m}_{\boldsymbol{\xi}}^{\ell-i+r}\left(\boldsymbol{M}_{k-1, \ell}+\boldsymbol{m}_{\boldsymbol{\varepsilon}}\right),
$$

for $k \in \mathbb{N}$ with $k \geqslant 2$ and $i \in\{1, \ldots, r\}$. This recursion implies

$$
\boldsymbol{X}_{r k-i+1}=\sum_{j=1}^{k} \boldsymbol{m}_{\xi}^{(k-j) r} \sum_{\ell=i}^{r} \boldsymbol{m}_{\boldsymbol{\xi}}^{\ell-i}\left(\boldsymbol{M}_{j, \ell}+\boldsymbol{m}_{\boldsymbol{\varepsilon}}\right)+\sum_{j=2}^{k} \boldsymbol{m}_{\boldsymbol{\xi}}^{(k-j) r} \sum_{\ell=1}^{i-1} \boldsymbol{m}_{\boldsymbol{\xi}}^{\ell-i+r}\left(\boldsymbol{M}_{j-1, \ell}+\boldsymbol{m}_{\boldsymbol{\varepsilon}}\right)
$$

for $k \in \mathbb{N}$ and $i \in\{1, \ldots, r\}$. Applying Lemma A.4, which is a version of the continuous mapping theorem, together with (3.10), (3.6) and Theorem 3.4, we show the following convergence result.

Theorem 3.6. Under the assumptions of Theorem 3.1, we have

$$
\boldsymbol{\mathcal { X }}^{(n)} \stackrel{\mathcal{D}}{\longrightarrow} \mathcal{X} \quad \text { as } n \rightarrow \infty,
$$

where $\mathcal{X}=\left(\mathcal{X}_{t}\right)_{t \in \mathbb{R}_{+}}$is given by (3.2) with

$$
\mathcal{Y}_{t}:=\Pi\left(\mathcal{N}_{t}+\frac{t}{r} \sum_{\ell=1}^{r} \boldsymbol{m}_{\xi}^{\ell-1} \boldsymbol{m}_{\varepsilon}\right), \quad t \in \mathbb{R}_{+},
$$

for which we have $\left(\boldsymbol{m}_{\xi}^{r} \mathcal{Y}_{t}\right)_{t \in \mathbb{R}_{+}}=\left(\mathcal{Y}_{t}\right)_{t \in \mathbb{R}_{+}}$almost surely.

Proof of Theorem 3.1. Theorem 3.1 is an easy consequence of Theorems 3.5 and 3.6. Indeed, $\boldsymbol{\Pi}=\boldsymbol{\Pi}_{1} \oplus \cdots \oplus \boldsymbol{\Pi}_{r}$ and $\boldsymbol{\Pi}_{i}=r \boldsymbol{u}_{i} \boldsymbol{v}_{i}^{\top}, r \boldsymbol{v}_{i}^{\top} \boldsymbol{u}_{i}=1$ for all $i \in\{1, \ldots, r\}$, hence we conclude from Theorems 3.5 and 3.6 that for each $i \in\{1, \ldots, r\}$, the process $\mathcal{Z}_{t, i}:=\boldsymbol{v}_{i}^{\top} \mathcal{Y}_{t, i}, t \in \mathbb{R}_{+}$, satisfies

$$
\mathcal{Z}_{t, i}=\boldsymbol{v}_{i}^{\top} \boldsymbol{\Pi}_{i}\left(\mathcal{N}_{t, i}+\frac{t}{r} \sum_{\ell=i}^{i+r-1} \widetilde{\boldsymbol{m}}_{i, \ell} \boldsymbol{m}_{\boldsymbol{\varepsilon}, \ell}\right)=\boldsymbol{v}_{i}^{\top}\left(\mathcal{N}_{t, i}+\frac{t}{r} \sum_{\ell=i}^{i+r-1} \widetilde{\boldsymbol{m}}_{i, \ell} \boldsymbol{m}_{\boldsymbol{\varepsilon}, \ell}\right), \quad t \in \mathbb{R}_{+},
$$


hence

$$
\mathcal{Z}_{t, i} \boldsymbol{u}_{i}=\boldsymbol{u}_{i} \boldsymbol{v}_{i}^{\top}\left(\mathcal{N}_{t, i}+\frac{t}{r} \sum_{\ell=i}^{i+r-1} \widetilde{\boldsymbol{m}}_{i, \ell} \boldsymbol{m}_{\boldsymbol{\varepsilon}, \ell}\right)=\boldsymbol{\Pi}_{i}\left(\mathcal{N}_{t, i}+\frac{t}{r} \sum_{\ell=i}^{i+r-1} \widetilde{\boldsymbol{m}}_{i, \ell} \boldsymbol{m}_{\boldsymbol{\varepsilon}, \ell}\right)=\mathcal{Y}_{t, i} .
$$

By Itô's formula, we obtain that $\left(\mathcal{Z}_{t, i}\right)_{t \in \mathbb{R}_{+}}$is a strong solution of the SDE

$$
\mathrm{d} \mathcal{Z}_{t, i}=r^{-1} \boldsymbol{v}_{i}^{\top} \sum_{\ell=i}^{i+r-1} \widetilde{\boldsymbol{m}}_{i, \ell} \boldsymbol{m}_{\boldsymbol{\varepsilon}, \ell} \mathrm{d} t+\boldsymbol{v}_{i}^{\top} \sqrt{r^{-1} \mathcal{Z}_{t, i}^{+}} \sum_{\ell=i}^{i+r-1} \widetilde{\boldsymbol{m}}_{i, \ell} \sqrt{\left(\widetilde{\boldsymbol{m}}_{\ell+1, i} \boldsymbol{u}_{i}\right) \odot \boldsymbol{V}_{\ell+1}} \mathrm{~d} \mathcal{W}_{t, \ell+i-1, \ell+1}
$$

with initial value $\mathcal{Z}_{0, i}=0$. This equation can be written in the form $(3.3)$, where $\left(\mathcal{W}_{t, i}\right)_{t \in \mathbb{R}_{+}}, i \in\{1, \ldots, r\}$, are independent standard Wiener processes. Indeed, we have

$$
\begin{aligned}
r \boldsymbol{v}_{i}^{\top} \boldsymbol{V}_{\boldsymbol{\xi}, \boldsymbol{\varepsilon}, i} \boldsymbol{v}_{i} & =\boldsymbol{v}_{i}^{\top} \sum_{\ell=i}^{i+r-1} \widetilde{\boldsymbol{m}}_{i, \ell}\left[\left(\widetilde{\boldsymbol{m}}_{\ell+1, i} \boldsymbol{u}_{i}\right) \odot \boldsymbol{V}_{\ell+1}\right] \widetilde{\boldsymbol{m}}_{i, \ell}^{\top} \boldsymbol{v}_{i} \\
& =\sum_{\ell=i}^{i+r-1}\left(\boldsymbol{v}_{i}^{\top} \widetilde{\boldsymbol{m}}_{i, \ell} \sqrt{\left(\widetilde{\boldsymbol{m}}_{\ell+1, i} \boldsymbol{u}_{i}\right) \odot \boldsymbol{V}_{\ell+1}}\right)\left(\boldsymbol{v}_{i}^{\top} \widetilde{\boldsymbol{m}}_{i, \ell} \sqrt{\left(\widetilde{\boldsymbol{m}}_{\ell+1, i} \boldsymbol{u}_{i}\right) \odot \boldsymbol{V}_{\ell+1}}\right)^{\top} \\
& =\sum_{\ell=i}^{i+r-1}\left\|\boldsymbol{v}_{i}^{\top} \widetilde{\boldsymbol{m}}_{i, \ell} \sqrt{\left(\widetilde{\boldsymbol{m}}_{\ell+1, i} \boldsymbol{u}_{i}\right) \odot \boldsymbol{V}_{\ell+1}}\right\|^{2}
\end{aligned}
$$

Hence, if $\boldsymbol{v}_{i}^{\top} \widetilde{\boldsymbol{m}}_{i, \ell} \sqrt{\left(\widetilde{\boldsymbol{m}}_{\ell+1, i} \boldsymbol{u}_{i}\right) \odot \boldsymbol{V}_{\ell+1}}=\mathbf{0}$ for each $\ell \in\{i, \ldots, i+r-1\}$, then (3.3) trivially follows, and if there exists $\ell \in\{i, \ldots, i+r-1\}$ with $\boldsymbol{v}_{i}^{\top} \widetilde{\boldsymbol{m}}_{i, \ell} \sqrt{\left(\widetilde{\boldsymbol{m}}_{\ell+1, i} \boldsymbol{u}_{i}\right) \odot \boldsymbol{V}_{\ell+1}} \neq \mathbf{0}$, then (3.3) holds with

$$
\mathcal{W}_{t, i}:=\frac{\boldsymbol{v}_{i}^{\top} \sum_{\ell=i}^{i+r-1} \widetilde{\boldsymbol{m}}_{i, \ell} \sqrt{\left(\widetilde{\boldsymbol{m}}_{\ell+1, i} \boldsymbol{u}_{i}\right) \odot \boldsymbol{V}_{\ell+1}} \mathcal{W}_{t, \ell+i-1, \ell+1}}{\boldsymbol{v}_{i}^{\top} \sum_{\ell=i}^{i+r-1} \widetilde{\boldsymbol{m}}_{i, \ell}\left[\left(\widetilde{\boldsymbol{m}}_{\ell+1, i} \boldsymbol{u}_{i}\right) \odot \boldsymbol{V}_{\ell+1}\right] \widetilde{\boldsymbol{m}}_{i, \ell}^{\top} \boldsymbol{v}_{i}}, \quad t \in \mathbb{R}_{+}, \quad i \in\{1, \ldots, r\}
$$

which are independent standard Wiener processes, since $\{(\ell+i-1, \ell+1): \ell \in\{i, \ldots, i+r-1\}\}, i \in\{1, \ldots, r\}$, are disjoint sets. Consequently, we conclude (3.1).

Remark 3.7. An alternative way of proving Theorem 3.1 is first checking that

$$
\boldsymbol{Y}_{k}:=\left[\begin{array}{c}
\boldsymbol{X}_{r k} \\
\boldsymbol{X}_{r k-1} \\
\vdots \\
\boldsymbol{X}_{r k-r+1}
\end{array}\right], \quad k \in \mathbb{Z}_{+},
$$

is a positively regular critical $r p$-type branching process with immigration (it can be done, for instance, by the help of generating functions), then determining the immigration mean vector and the offspring variation matrices of $\left(\boldsymbol{Y}_{k}\right)_{k \in \mathbb{Z}_{+}}$, and then applying the convergence result of Ispány and Pap [8]. This would have been also a cumbersome calculation.

\section{Proof of Theorem 3.5}

If $\left(\mathcal{M}_{t}\right)_{t \in \mathbb{R}_{+}}$is a strong solution of the $\operatorname{SDE}(3.5)$, then the process $\left(\mathcal{N}_{t}\right)_{t \in \mathbb{R}_{+}}$is a strong solution of the $\operatorname{SDE}(3.7)$ with initial value $\boldsymbol{\mathcal { N }}_{0}=\mathbf{0}$, and (3.8) trivially holds.

Using the block matrix form of $\boldsymbol{m}_{\boldsymbol{\xi}}, \boldsymbol{\Pi}$ and $\boldsymbol{V}_{\boldsymbol{\xi}_{1}}, \ldots, \boldsymbol{V}_{\boldsymbol{\xi}_{p}}$ (see (2.1), (2.2) and (2.4)), we obtain

$$
\mathrm{d} \mathcal{N}_{t, i}=\frac{1}{r} \sum_{j=1}^{r} \widetilde{\boldsymbol{m}}_{i, i+j-1} \sqrt{\left[\widetilde{\boldsymbol{m}}_{i-r+j, i} \boldsymbol{\Pi}_{i}\left(r \mathcal{N}_{t, i}+t \sum_{\ell=i}^{i+r-1} \widetilde{\boldsymbol{m}}_{i, \ell} \boldsymbol{m}_{\boldsymbol{\varepsilon}, \ell}\right)\right]^{+} \odot \boldsymbol{V}_{i-r+j}} \mathrm{~d} \mathcal{W}_{t, j, i+j}
$$


for each $i \in\{1, \ldots, r\}$. Indeed, the covariance matrices $\boldsymbol{V}_{\boldsymbol{\xi}_{j}} j \in\{1, \ldots, p\}$, have block-diagonal form, see (2.4), hence

$$
\begin{aligned}
\sqrt{\left[\boldsymbol{m}_{\boldsymbol{\xi}}^{r-j} \boldsymbol{\Pi}\left(r \mathcal{N}_{t}+t \sum_{\ell=1}^{r} \boldsymbol{m}_{\boldsymbol{\xi}}^{\ell-1} \boldsymbol{m}_{\boldsymbol{\varepsilon}}\right)\right]^{+} \odot \boldsymbol{V}_{\boldsymbol{\xi}}} \\
=\bigoplus_{i=r-j+2}^{2 r-j+1} \sqrt{\left[\widetilde{\boldsymbol{m}}_{i-r+j, i} \boldsymbol{\Pi}_{i}\left(r \mathcal{N}_{t, i}+t \sum_{\ell=i}^{r+i-1} \widetilde{\boldsymbol{m}}_{i, \ell} \boldsymbol{m}_{\boldsymbol{\varepsilon}, \ell}\right)\right]^{+} \odot \boldsymbol{V}_{i-r+j}},
\end{aligned}
$$

where we also used that for an arbitrary matrix $\boldsymbol{A} \in \mathbb{R}^{p \times p}$ with partitioning

$$
\boldsymbol{A}=\left[\begin{array}{ccc}
\boldsymbol{A}_{1,1} & \cdots & \boldsymbol{A}_{1, r} \\
\vdots & \ddots & \vdots \\
\boldsymbol{A}_{r, 1} & \cdots & \boldsymbol{A}_{r, r}
\end{array}\right]
$$

with respect to the partitioning $D_{1}, \ldots, D_{r}$ of the coordinates $\{1, \ldots, p\}$, we have

$$
\boldsymbol{m}_{\boldsymbol{\xi}}^{k} \boldsymbol{A}=\left[\begin{array}{cccc}
\widetilde{\boldsymbol{m}}_{1, k+1} \boldsymbol{A}_{k+1,1} & \cdots & \widetilde{\boldsymbol{m}}_{1, k+1} \boldsymbol{A}_{k+1, r} \\
\vdots & \ddots & \vdots \\
\widetilde{\boldsymbol{m}}_{r, k+r} \boldsymbol{A}_{k+r, 1} & \cdots & \widetilde{\boldsymbol{m}}_{r, k+r} \boldsymbol{A}_{k+r, r}
\end{array}\right]
$$

for all $k \in\{1, \ldots r-1\}$, where the first subscript of $\boldsymbol{A}_{i, j}$ is considered modulo $r$. Substituting this into (3.7) and using again the above block form of $\boldsymbol{m}_{\xi}^{k} \boldsymbol{A}$ for $\boldsymbol{A} \in \mathbb{R}^{p \times p}$ and $k \in\{1, \ldots r-1\}$, we obtain (4.1). Using $\boldsymbol{\Pi}_{i}=r \boldsymbol{u}_{i} \boldsymbol{v}_{i}^{\top}$ for all $i \in\{1, \ldots, r\}$, equation (4.1) can be written in the form (3.9).

\section{Proof of Theorem 3.4}

In order to prove $\mathcal{M}^{(n)} \stackrel{\mathcal{D}}{\longrightarrow} \mathcal{M}$, we want to apply Theorem A.3 for $\mathcal{U}=\mathcal{M}, \boldsymbol{U}_{k}^{(n)}=n^{-1} \boldsymbol{M}_{k}$ and $\mathcal{F}_{k}^{(n)}:=\mathcal{F}_{k}$ for $n \in \mathbb{N}$ and $k \in \mathbb{Z}_{+}$, and with coefficient function $\gamma: \mathbb{R}_{+} \times\left(\mathbb{R}^{p}\right)^{r} \rightarrow\left(\mathbb{R}^{p \times p}\right)^{r \times r}$ of the SDE (3.5) given by

$$
\gamma(t, \boldsymbol{x})=\frac{1}{r} \bigoplus_{i=1}^{r} \sqrt{\left[\boldsymbol{m}_{\boldsymbol{\xi}}^{r-i} \boldsymbol{\Pi} \sum_{j=1}^{r} \boldsymbol{m}_{\boldsymbol{\xi}}^{j-1}\left(r \boldsymbol{x}_{j}+t \boldsymbol{m}_{\boldsymbol{\varepsilon}}\right)\right]^{+} \odot \boldsymbol{V}_{\boldsymbol{\xi}}}, \quad \boldsymbol{x}=\left[\begin{array}{c}
\boldsymbol{x}_{1} \\
\vdots \\
\boldsymbol{x}_{r}
\end{array}\right] \in\left(\mathbb{R}^{p}\right)^{r} .
$$

The aim of the following discussion is to show that the $\operatorname{SDE}(3.5)$ has a unique strong solution $\left(\mathcal{M}_{t}^{\left(x_{0}\right)}\right)_{t \in \mathbb{R}_{+}}$ with initial value $\mathcal{M}_{0}^{\left(\boldsymbol{x}_{0}\right)}=\boldsymbol{x}_{0}$ for all $\boldsymbol{x}_{0} \in\left(\mathbb{R}^{p}\right)^{r}$. Clearly, it is sufficient to prove that the SDE (3.7) has a unique strong solution $\left(\mathcal{N}_{t}^{\left(\boldsymbol{y}_{0}\right)}\right)_{t \in \mathbb{R}_{+}}$with initial value $\mathcal{N}_{0}^{\left(\boldsymbol{y}_{0}\right)}=\boldsymbol{y}_{0}$ for all $\boldsymbol{y}_{0} \in\left(\mathbb{R}^{p}\right)^{r}$. Indeed, if $\left(\mathcal{M}_{t}^{\left(\boldsymbol{x}_{0}\right)}\right)_{t \in \mathbb{R}_{+}}$is a strong solution of the $\operatorname{SDE}(3.5)$ with initial value $\mathcal{M}_{0}^{\left(\boldsymbol{x}_{0}\right)}=\boldsymbol{x}_{0}$ with some

$$
\boldsymbol{x}_{0}=\left[\begin{array}{c}
\boldsymbol{x}_{0,1} \\
\vdots \\
\boldsymbol{x}_{0, r}
\end{array}\right] \in\left(\mathbb{R}^{p}\right)^{r}
$$

then $\mathcal{N}_{t}:=\sum_{i=1}^{r} \boldsymbol{m}_{\boldsymbol{\xi}}^{i-1} \mathcal{M}_{t, i}^{\left(\boldsymbol{x}_{0}\right)}$ is a strong solution of the $\operatorname{SDE}$ (3.7) with initial value $\sum_{i=1}^{r} \boldsymbol{m}_{\boldsymbol{\xi}}^{i-1} \boldsymbol{x}_{0, i}$. Conversely, if $\left(\mathcal{N}_{t}^{\left(\boldsymbol{y}_{0}\right)}\right)_{t \in \mathbb{R}_{+}}$is a strong solution of the $\operatorname{SDE}(3.7)$ with initial value $\boldsymbol{\mathcal { N }}_{0}^{\left(\boldsymbol{y}_{0}\right)}=\boldsymbol{y}_{0}$ with some $\boldsymbol{y}_{0} \in \mathbb{R}^{p}$ then with

$$
\boldsymbol{x}_{0}=\left[\begin{array}{c}
\boldsymbol{x}_{0,1} \\
\boldsymbol{x}_{0,2} \\
\vdots \\
\boldsymbol{x}_{0, r}
\end{array}\right]:=\left[\begin{array}{c}
\boldsymbol{y}_{0} \\
\mathbf{0} \\
\vdots \\
\mathbf{0}
\end{array}\right],
$$


we have $\boldsymbol{y}_{0}=\sum_{i=1}^{r} \boldsymbol{m}_{\boldsymbol{\xi}}^{i-1} \boldsymbol{x}_{0, i} \in \mathbb{R}^{p}$, and

$$
\mathcal{M}_{t, i}:=\boldsymbol{x}_{0, i}+\frac{1}{r} \int_{0}^{t} \sqrt{\left[\boldsymbol{m}_{\boldsymbol{\xi}}^{r-i} \boldsymbol{\Pi}\left(r \mathcal{N}_{s}^{\left(\boldsymbol{y}_{0}\right)}+s \sum_{\ell=1}^{r} \boldsymbol{m}_{\boldsymbol{\xi}}^{\ell-1} \boldsymbol{m}_{\boldsymbol{\varepsilon}}\right)\right]^{+} \odot \boldsymbol{V}_{\boldsymbol{\xi}}} \mathrm{d} \mathcal{W}_{s, i}, \quad i \in\{1, \ldots, r\},
$$

is a strong solution of the $\operatorname{SDE}(3.5)$ with initial value $\boldsymbol{x}_{0}$.

Hence it is enough to show that the $\operatorname{SDE}(3.7)$ has a unique strong solution $\left(\mathcal{N}_{t}^{\left(\boldsymbol{y}_{0}\right)}\right)_{t \in \mathbb{R}_{+}}$with initial value $\mathcal{N}_{0}^{\left(\boldsymbol{y}_{0}\right)}=\boldsymbol{y}_{0}$ for all $\boldsymbol{y}_{0} \in \mathbb{R}^{p}$. First observe that if $\left(\mathcal{N}_{t, i}^{\left(\boldsymbol{y}_{0, i}\right)}\right)_{t \in \mathbb{R}_{+}}$is a strong solution of the SDE (3.9) with initial value $\mathcal{N}_{0, i}^{\left(\boldsymbol{y}_{0, i}\right)}=\boldsymbol{y}_{0, i} \in \mathbb{R}^{\left|D_{i}\right|}$, then, by Itô's formula, the process $\left(\mathcal{P}_{t, i}, \mathcal{Q}_{t, i}\right)_{t \in \mathbb{R}_{+}}$, defined by

$$
\mathcal{P}_{t, i}:=\boldsymbol{v}_{i}^{\top}\left(\mathcal{N}_{t, i}^{\left(\boldsymbol{y}_{0, i}\right)}+\frac{t}{r} \sum_{\ell=i}^{i+r-1} \widetilde{\boldsymbol{m}}_{i, \ell} \boldsymbol{m}_{\boldsymbol{\varepsilon}, \ell}\right), \quad \mathcal{Q}_{t, i}:=\mathcal{N}_{t, i}^{\left(\boldsymbol{y}_{0, i}\right)}-\mathcal{P}_{t, i} \boldsymbol{u}_{i}
$$

is a strong solution of the SDE

$$
\left\{\begin{aligned}
\mathrm{d} \mathcal{P}_{t, i}= & \frac{1}{r} \boldsymbol{v}_{i}^{\top} \sum_{\ell=i}^{i+r-1} \widetilde{\boldsymbol{m}}_{i, \ell} \boldsymbol{m}_{\boldsymbol{\varepsilon}, \ell} \mathrm{d} t \\
& +\sqrt{r^{-1} \mathcal{P}_{t, i}^{+}} \boldsymbol{v}_{i}^{\top} \sum_{\ell=i}^{i+r-1} \widetilde{\boldsymbol{m}}_{i, \ell} \sqrt{\left(\widetilde{\boldsymbol{m}}_{\ell+1, i+r} \boldsymbol{u}_{i}\right) \odot \boldsymbol{V}_{\ell+1}} \mathrm{~d} \mathcal{W}_{t, \ell+i-1, \ell+1}, \\
\mathrm{~d} \mathcal{Q}_{t, i}= & -\frac{1}{r} \boldsymbol{\Pi}_{i} \sum_{\ell=i}^{i+r-1} \widetilde{\boldsymbol{m}}_{i, \ell} \boldsymbol{m}_{\boldsymbol{\varepsilon}, \ell} \mathrm{d} t \\
& +\sqrt{r^{-1} \mathcal{P}_{t, i}^{+}}\left(\boldsymbol{I}_{\left|D_{i}\right|}-\boldsymbol{\Pi}_{i}\right) \sum_{\ell=i}^{i+r-1} \widetilde{\boldsymbol{m}}_{i, \ell} \sqrt{\left(\widetilde{\boldsymbol{m}}_{\ell+1, i+r} \boldsymbol{u}_{i}\right) \odot \boldsymbol{V}_{\ell+1}} \mathrm{~d} \mathcal{W}_{t, \ell+i-1, \ell+1}
\end{aligned}\right.
$$

with initial value $\left(\mathcal{P}_{0, i}, \mathcal{Q}_{0, i}\right)=\left(\boldsymbol{v}_{i}^{\top} \boldsymbol{y}_{0, i},\left(\boldsymbol{I}_{\left|D_{i}\right|}-\boldsymbol{\Pi}_{i}\right) \boldsymbol{y}_{0, i}\right)$. Indeed, the first SDE of (5.1) is an easy consequence of the SDE (3.9). The second one can be checked as follows. By Itô's formula,

$$
\begin{aligned}
\mathrm{d} \mathcal{Q}_{t, i} & =\mathrm{d} \mathcal{N}_{t, i}^{\left(\boldsymbol{y}_{0, i}\right)}-\boldsymbol{u}_{i} \mathrm{~d} \mathcal{P}_{t, i}=\mathrm{d} \mathcal{N}_{t, i}^{\left(\boldsymbol{y}_{0, i}\right)}-\boldsymbol{u}_{i} \boldsymbol{v}_{i}^{\top}\left(\mathrm{d} \mathcal{N}_{t, i}^{\left(\boldsymbol{y}_{0, i}\right)}+\frac{1}{r} \sum_{\ell=i}^{i+r-1} \widetilde{\boldsymbol{m}}_{i, \ell} \boldsymbol{m}_{\boldsymbol{\varepsilon}, \ell} \mathrm{d} t\right) \\
& =-\frac{1}{r} \boldsymbol{\Pi}_{i} \sum_{\ell=i}^{i+r-1} \widetilde{\boldsymbol{m}}_{i, \ell} \boldsymbol{m}_{\boldsymbol{\varepsilon}, \ell} \mathrm{d} t+\left(\boldsymbol{I}_{\left|D_{i}\right|}-\boldsymbol{\Pi}_{i}\right) \mathrm{d} \mathcal{N}_{t, i}^{\left(\boldsymbol{y}_{0, i}\right)}
\end{aligned}
$$

with $\mathcal{Q}_{0, i}=\mathcal{N}_{0, i}^{\left(\boldsymbol{y}_{0, i}\right)}-\mathcal{P}_{0, i} \boldsymbol{u}_{i}=\boldsymbol{y}_{0, i}-\left(\boldsymbol{v}_{i}^{\top} \boldsymbol{y}_{0, i}\right) \boldsymbol{u}_{i}=\boldsymbol{y}_{0, i}-\boldsymbol{u}_{i} \boldsymbol{v}_{i}^{\top} \boldsymbol{y}_{0, i}=\left(\boldsymbol{I}_{\left|D_{i}\right|}-\boldsymbol{\Pi}_{i}\right) \boldsymbol{y}_{0, i}$. Conversely, if $\left(\mathcal{P}_{t, i}^{\left(p_{0, i}, \boldsymbol{q}_{0, i}\right)}, \mathcal{Q}_{t, i}^{\left(p_{0, i}, \boldsymbol{q}_{0, i}\right)}\right)_{t \in \mathbb{R}_{+}}$is a strong solution of the $\operatorname{SDE}(5.1)$ with initial value $\left(\mathcal{P}_{0, i}^{\left(p_{0, i}, \boldsymbol{q}_{0, i}\right)}, \mathcal{Q}_{0, i}^{\left(p_{0, i}, \boldsymbol{q}_{0, i}\right)}\right)=$ $\left(p_{0, i}, \boldsymbol{q}_{0, i}\right) \in \mathbb{R} \times \mathbb{R}^{\left|D_{i}\right|}$, then, again by Itô's formula,

$$
\mathcal{N}_{t, i}:=\mathcal{P}_{t, i}^{\left(p_{0, i}, \boldsymbol{q}_{0, i}\right)} \boldsymbol{u}_{i}+\mathcal{Q}_{t, i}^{\left(p_{0, i}, \boldsymbol{q}_{0, i}\right)}, \quad t \in \mathbb{R}_{+},
$$

is a strong solution of the $\operatorname{SDE}(3.9)$ with initial value $\mathcal{N}_{0, i}=p_{0, i} \boldsymbol{u}_{i}+\boldsymbol{q}_{0, i}$. The correspondence $\boldsymbol{y}_{0, i} \leftrightarrow$ $\left(p_{0, i}, \boldsymbol{q}_{0, i}\right):=\left(\boldsymbol{v}_{i}^{\top} \boldsymbol{y}_{0, i},\left(\boldsymbol{I}_{\left|D_{i}\right|}-\boldsymbol{\Pi}_{i}\right) \boldsymbol{y}_{0, i}\right)$ is a bijection between $\mathbb{R}^{\left|D_{i}\right|}$ and $\mathbb{R} \times\left\{\boldsymbol{q} \in \mathbb{R}^{\left|D_{i}\right|}: \boldsymbol{v}_{i}^{\top} \boldsymbol{q}=0\right\}$, since $\boldsymbol{y}_{0, i}=p_{0, i} \boldsymbol{u}_{i}+\boldsymbol{q}_{0, i}$, and

$$
\left(\boldsymbol{I}_{\left|D_{i}\right|}-\boldsymbol{\Pi}_{i}\right)\left(p_{0, i} \boldsymbol{u}_{i}+\boldsymbol{q}_{0, i}\right)=p_{0, i} \boldsymbol{u}_{i}+\boldsymbol{q}_{0, i}-\boldsymbol{\Pi}_{i} p_{0, i} \boldsymbol{u}_{i}+\boldsymbol{\Pi}_{i} \boldsymbol{q}_{0, i}=p_{0, i} \boldsymbol{u}_{i}+\boldsymbol{q}_{0, i}-p_{0, i} \boldsymbol{u}_{i} \boldsymbol{v}_{i}^{\top} \boldsymbol{u}_{i}+\boldsymbol{u}_{i} \boldsymbol{v}_{i}^{\top} \boldsymbol{q}_{0, i}=\boldsymbol{q}_{0, i} .
$$

The right hand side of the SDE (5.1) contains only the process $\left(\mathcal{P}_{t, i}\right)_{t \in \mathbb{R}_{+}}$, hence it is enough to show that the first equation of (5.1) has a unique strong solution $\left(\mathcal{P}_{t, i}^{\left(p_{0, i}, \boldsymbol{q}_{0, i}\right)}\right)_{t \in \mathbb{R}_{+}}$with initial value $\mathcal{P}_{0, i}^{\left(p_{0, i}, \boldsymbol{q}_{0, i}\right)}=p_{0, i}$ for all $p_{0, i} \in \mathbb{R}$. The first equation of (5.1) is the same as (3.11), which can be written in the form (3.3), see the end of 
Section 3. Hence, by Remark 3.2, the first equation of the $\operatorname{SDE}(5.1)$ has a unique strong solution $\left(\mathcal{P}_{t, i}^{\left(p_{0, i}\right)}\right)_{t \in \mathbb{R}_{+}}$ with initial value $\mathcal{P}_{0, i}^{\left(p_{0, i}\right)}=p_{0, i}$ for all $p_{0, i} \in \mathbb{R}$. Consequently, the $\operatorname{SDE}$ (5.1), and hence the SDE (3.5) admit a unique strong solution with arbitrary initial value.

Now we show that conditions (i) and (ii) of Theorem A.3 hold. We have to check that for each $T>0$,

$$
\begin{aligned}
& \sup _{t \in[0, T]}\left\|\frac{1}{(r n)^{2}} \sum_{k=1}^{\lfloor n t\rfloor} \mathbb{E}\left[\boldsymbol{M}_{k} \boldsymbol{M}_{k}^{\top} \mid \mathcal{F}_{r k-r}\right]-\int_{0}^{t}\left(\mathcal{R}_{s}^{(n)}\right)^{+} \mathrm{d} s\right\| \stackrel{\mathbb{P}}{\longrightarrow} 0, \\
& \frac{1}{(r n)^{2}} \sum_{k=1}^{\lfloor n T\rfloor} \mathbb{E}\left(\left\|\boldsymbol{M}_{k}\right\|^{2} \mathbb{1}_{\left\{\left\|\boldsymbol{M}_{k}\right\|>n \theta\right\}} \mid \mathcal{F}_{r k-r}\right) \stackrel{\mathbb{P}}{\longrightarrow} 0, \quad \text { for all } \theta>0,
\end{aligned}
$$

as $n \rightarrow \infty$, where the process $\left(\boldsymbol{\mathcal { R }}_{s}^{(n)}\right)_{s \in \mathbb{R}_{+}}$is defined by

$$
\mathcal{R}_{s}^{(n)}:=\frac{1}{r^{2}} \bigoplus_{i=1}^{r}\left\{\left[\boldsymbol{m}_{\boldsymbol{\xi}}^{r-i} \boldsymbol{\Pi} \sum_{j=1}^{r} \boldsymbol{m}_{\boldsymbol{\xi}}^{j-1}\left(\mathcal{M}_{s, j}^{(n)}+r^{-1} s \boldsymbol{m}_{\boldsymbol{\varepsilon}}\right)\right] \odot \boldsymbol{V}_{\boldsymbol{\xi}}\right\}
$$

for $s \in \mathbb{R}_{+}, n \in \mathbb{N}$. By (3.4),

$$
\begin{aligned}
\boldsymbol{\Pi} \sum_{j=1}^{r} \boldsymbol{m}_{\boldsymbol{\xi}}^{j-1}\left(\boldsymbol{M}_{s, j}^{(n)}+r^{-1} s \boldsymbol{m}_{\boldsymbol{\varepsilon}}\right) \\
=\boldsymbol{\Pi} \sum_{j=1}^{r} \boldsymbol{m}_{\boldsymbol{\xi}}^{j-1}\left((r n)^{-1} \sum_{k=1}^{\lfloor n s\rfloor}\left(\boldsymbol{X}_{r k-j+1}-\boldsymbol{m}_{\boldsymbol{\xi}} \boldsymbol{X}_{r k-j}-\boldsymbol{m}_{\boldsymbol{\varepsilon}}\right)+r^{-1} s \boldsymbol{m}_{\boldsymbol{\varepsilon}}\right) \\
=(r n)^{-1} \boldsymbol{\Pi} \sum_{k=1}^{\lfloor n s\rfloor} \sum_{j=1}^{r}\left(\boldsymbol{m}_{\boldsymbol{\xi}}^{j-1} \boldsymbol{X}_{r k-j+1}-\boldsymbol{m}_{\boldsymbol{\xi}}^{j} \boldsymbol{X}_{r k-j}-\boldsymbol{m}_{\boldsymbol{\xi}}^{j-1} \boldsymbol{m}_{\boldsymbol{\varepsilon}}\right)+r^{-1} s \boldsymbol{\Pi} \sum_{j=1}^{r} \boldsymbol{m}_{\boldsymbol{\xi}}^{j-1} \boldsymbol{m}_{\boldsymbol{\varepsilon}} \\
=(r n)^{-1} \boldsymbol{\Pi} \sum_{k=1}^{\lfloor n s\rfloor}\left(\boldsymbol{X}_{r k}-\boldsymbol{m}_{\boldsymbol{\xi}}^{r} \boldsymbol{X}_{r k-r}-\sum_{j=1}^{r} \boldsymbol{m}_{\boldsymbol{\xi}}^{j-1} \boldsymbol{m}_{\varepsilon}\right)+r^{-1} s \boldsymbol{\Pi} \sum_{j=1}^{r} \boldsymbol{m}_{\boldsymbol{\xi}}^{j-1} \boldsymbol{m}_{\boldsymbol{\varepsilon}} \\
=(r n)^{-1} \sum_{k=1}^{\lfloor n s\rfloor}\left(\boldsymbol{\Pi} \boldsymbol{X}_{r k}-\boldsymbol{\Pi} \boldsymbol{X}_{r k-r}-\boldsymbol{\Pi} \sum_{j=1}^{r} \boldsymbol{m}_{\boldsymbol{\xi}}^{j-1} \boldsymbol{m}_{\boldsymbol{\varepsilon}}\right)+r^{-1} s \boldsymbol{\Pi} \sum_{j=1}^{r} \boldsymbol{m}_{\boldsymbol{\xi}}^{j-1} \boldsymbol{m}_{\boldsymbol{\varepsilon}} \\
=(r n)^{-1} \boldsymbol{\Pi} \boldsymbol{X}_{r\lfloor n s\rfloor}+\left(s-\frac{\lfloor n s\rfloor}{n}\right) r^{-1} \boldsymbol{\Pi} \sum_{j=1}^{r} \boldsymbol{m}_{\boldsymbol{\xi}}^{j-1} \boldsymbol{m}_{\boldsymbol{\varepsilon}}
\end{aligned}
$$

where we used

$$
\boldsymbol{\Pi} \boldsymbol{m}_{\boldsymbol{\xi}}^{r}=\left(\lim _{n \rightarrow \infty} \boldsymbol{m}_{\xi}^{n r}\right) \boldsymbol{m}_{\boldsymbol{\xi}}^{r}=\lim _{n \rightarrow \infty} \boldsymbol{m}_{\xi}^{(n+1) r}=\boldsymbol{\Pi}
$$

Consequently,

$$
\boldsymbol{\mathcal { R }}_{s}^{(n)}=\frac{1}{r^{2}} \bigoplus_{i=1}^{r}\left\{\left[n^{-1} \boldsymbol{m}_{\boldsymbol{\xi}}^{r-i} \boldsymbol{\Pi} \boldsymbol{X}_{r\lfloor n s\rfloor}+\left(s-\frac{\lfloor n s\rfloor}{n}\right) \boldsymbol{\Pi} \sum_{j=1}^{r} \boldsymbol{m}_{\boldsymbol{\xi}}^{j-1} \boldsymbol{m}_{\boldsymbol{\varepsilon}}\right] \odot \boldsymbol{V}_{\boldsymbol{\xi}}\right\}
$$

since

$$
\boldsymbol{m}_{\boldsymbol{\xi}}^{r-i} \boldsymbol{\Pi}=\boldsymbol{m}_{\xi}^{r-i}\left(\lim _{n \rightarrow \infty} \boldsymbol{m}_{\xi}^{n r}\right)=\left(\lim _{n \rightarrow \infty} \boldsymbol{m}_{\xi}^{n r+r-i}\right)=\left(\lim _{n \rightarrow \infty} \boldsymbol{m}_{\xi}^{n r}\right) \boldsymbol{m}_{\xi}^{r-i}=\boldsymbol{\Pi} \boldsymbol{m}_{\xi}^{r-i}
$$

and (5.4) implies

$$
\boldsymbol{m}_{\boldsymbol{\xi}}^{r-i} \boldsymbol{\Pi} \sum_{j=1}^{r} \boldsymbol{m}_{\boldsymbol{\xi}}^{j-1}=\boldsymbol{\Pi} \boldsymbol{m}_{\boldsymbol{\xi}}^{r-i} \sum_{j=1}^{r} \boldsymbol{m}_{\boldsymbol{\xi}}^{j-1}=\boldsymbol{\Pi}\left(\sum_{j=1}^{i} \boldsymbol{m}_{\boldsymbol{\xi}}^{j-1+r-i}+\boldsymbol{m}_{\boldsymbol{\xi}}^{r} \sum_{j=i+1}^{r} \boldsymbol{m}_{\boldsymbol{\xi}}^{j-1-i}\right)=\boldsymbol{\Pi} \sum_{j=1}^{r} \boldsymbol{m}_{\boldsymbol{\xi}}^{j-1} .
$$


Thus $\left(\boldsymbol{\mathcal { R }}_{t}^{(n)}\right)^{+}=\boldsymbol{\mathcal { R }}_{t}^{(n)}$, and

$$
\begin{aligned}
\int_{0}^{t}\left(\mathcal{R}_{s}^{(n)}\right)^{+} \mathrm{d} s= & \bigoplus_{i=1}^{r}\left\{\left[\frac{1}{(n r)^{2}} \boldsymbol{m}_{\boldsymbol{\xi}}^{r-i} \boldsymbol{\Pi} \sum_{\ell=0}^{\lfloor n t\rfloor-1} \boldsymbol{X}_{r \ell}+\frac{n t-\lfloor n t\rfloor}{(n r)^{2}} \boldsymbol{m}_{\boldsymbol{\xi}}^{r-i} \boldsymbol{\Pi} \boldsymbol{X}_{r\lfloor n t\rfloor}\right.\right. \\
& \left.\left.+\frac{\lfloor n t\rfloor+(n t-\lfloor n t\rfloor)^{2}}{2(n r)^{2}} \boldsymbol{\Pi} \sum_{j=1}^{r} \boldsymbol{m}_{\boldsymbol{\xi}}^{j-1} \boldsymbol{m}_{\boldsymbol{\varepsilon}}\right] \odot \boldsymbol{V}_{\boldsymbol{\xi}}\right\} .
\end{aligned}
$$

Using (A.3), we obtain

$$
\frac{1}{(n r)^{2}} \sum_{k=1}^{\lfloor n t\rfloor} \mathbb{E}\left(\boldsymbol{M}_{k} \boldsymbol{M}_{k}^{\top} \mid \mathcal{F}_{r k-r}\right)=\bigoplus_{i=1}^{r}\left\{\frac{\lfloor n t\rfloor}{n^{2}} \boldsymbol{V}_{\boldsymbol{\varepsilon}}+\frac{1}{n^{2}} \sum_{k=1}^{\lfloor n t\rfloor}\left[\boldsymbol{m}_{\boldsymbol{\xi}}^{r-i} \boldsymbol{X}_{r k-r}+\sum_{j=1}^{r-i} \boldsymbol{m}_{\boldsymbol{\xi}}^{j-1} \boldsymbol{m}_{\boldsymbol{\varepsilon}}\right] \odot \boldsymbol{V}_{\boldsymbol{\xi}}\right\}
$$

Hence, taking into account that $\boldsymbol{X}_{0}=\mathbf{0}$, in order to show (5.2), it suffices to prove

$$
\left.\frac{1}{n^{2}} \sup _{t \in[0, T]} \sum_{k=1}^{L} n t\right\rfloor\left\|\left(\boldsymbol{I}_{p}-\boldsymbol{\Pi}\right) \boldsymbol{X}_{r k}\right\| \stackrel{\mathbb{P}}{\longrightarrow} 0, \quad \frac{1}{n^{2}} \sup _{t \in[0, T]}\left\|\boldsymbol{X}_{r\lfloor n t\rfloor}\right\| \stackrel{\mathbb{P}}{\longrightarrow} 0
$$

as $n \rightarrow \infty$. Using (3.10) and (5.4), we obtain

$$
\left(\boldsymbol{I}_{p}-\boldsymbol{\Pi}\right) \boldsymbol{X}_{r k}=\left(\boldsymbol{I}_{p}-\boldsymbol{\Pi}\right) \sum_{j=1}^{k} \boldsymbol{m}_{\boldsymbol{\xi}}^{(k-j) r} \sum_{i=1}^{r} \boldsymbol{m}_{\boldsymbol{\xi}}^{i-1}\left(\boldsymbol{M}_{j, i}+\boldsymbol{m}_{\boldsymbol{\varepsilon}}\right)=\sum_{j=1}^{k}\left(\boldsymbol{m}_{\boldsymbol{\xi}}^{(k-j) r}-\boldsymbol{\Pi}\right) \sum_{i=1}^{r} \boldsymbol{m}_{\boldsymbol{\xi}}^{i-1}\left(\boldsymbol{M}_{j, i}+\boldsymbol{m}_{\boldsymbol{\varepsilon}}\right) .
$$

Hence by (2.3),

$$
\begin{aligned}
& \left.\sum_{k=1}^{\lfloor n t\rfloor}\left\|\left(\boldsymbol{I}_{p}-\boldsymbol{\Pi}\right) \boldsymbol{X}_{r k}\right\| \leqslant c \sum_{k=1}^{\llcorner} n t\right\rfloor \sum_{j=1}^{k} \kappa^{k-j} \sum_{i=1}^{r}\left\|\boldsymbol{m}_{\boldsymbol{\xi}}^{i-1}\right\|\left\|\boldsymbol{M}_{j, i}+\boldsymbol{m}_{\boldsymbol{\varepsilon}}\right\| \\
& \left.\left.\leqslant c K \sum_{j=1}^{\llcorner} n t\right\rfloor \sum_{k=j}^{\llcorner} n t\right\rfloor \kappa^{k-j}\left(\sum_{i=1}^{r}\left\|\boldsymbol{M}_{j, i}\right\|+r\left\|\boldsymbol{m}_{\boldsymbol{\varepsilon}}\right\|\right) \leqslant \frac{c K}{1-\kappa}\left(\sum_{j=1}^{\lfloor n t\rfloor} \sum_{i=1}^{r}\left\|\boldsymbol{M}_{j, i}\right\|+r\lfloor n t\rfloor\left\|\boldsymbol{m}_{\boldsymbol{\varepsilon}}\right\|\right),
\end{aligned}
$$

where $K:=\max _{i \in\{1, \ldots, r\}}\left\|\boldsymbol{m}_{\boldsymbol{\xi}}^{i-1}\right\|$. Moreover, by (3.10),

$$
\left\|\boldsymbol{X}_{r\lfloor n t\rfloor}\right\| \leqslant \sum_{j=1}^{\lfloor n t\rfloor}\left\|\boldsymbol{m}_{\xi}^{(\lfloor n t\rfloor-j) r}\right\| \sum_{i=1}^{r}\left\|\boldsymbol{m}_{\xi}^{i-1}\right\|\left\|\boldsymbol{M}_{j, i}+\boldsymbol{m}_{\boldsymbol{\varepsilon}}\right\| \leqslant K(c+\|\boldsymbol{\Pi}\|)\left(r\lfloor n t\rfloor\left\|\boldsymbol{m}_{\boldsymbol{\varepsilon}}\right\|+\sum_{j=1}^{\lfloor n t\rfloor} \sum_{i=1}^{r}\left\|\boldsymbol{M}_{j, i}\right\|\right),
$$

since $\left\|\boldsymbol{m}_{\xi}^{(\lfloor n t\rfloor-j) r}\right\| \leqslant\left\|\boldsymbol{m}_{\boldsymbol{\xi}}^{(\lfloor n t\rfloor-j) r}-\boldsymbol{\Pi}\right\|+\|\boldsymbol{\Pi}\| \leqslant c+\|\boldsymbol{\Pi}\|$ by (2.3). Consequently, in order to prove (5.5), it suffices to show

$$
\frac{1}{n^{2}} \sum_{j=1}^{\lfloor n T\rfloor}\left\|\boldsymbol{M}_{j, i}\right\| \stackrel{\mathbb{P}}{\longrightarrow} 0 \quad \text { as } n \rightarrow \infty \text { for all } i \in\{1, \ldots, r\} .
$$

In fact, Lemma A.2 yields $n^{-2} \sum_{j=1}^{\lfloor n T\rfloor} \mathbb{E}\left(\left\|\boldsymbol{M}_{j, i}\right\|\right) \rightarrow 0, i \in\{1, \ldots, r\}$, thus we obtain (5.2).

To prove (5.3), consider the decomposition $\boldsymbol{M}_{k}=\boldsymbol{N}_{k}+\left(\boldsymbol{\delta}_{k}-\mathbb{E}\left(\boldsymbol{\delta}_{k}\right)\right)$, where, by (A.5),

$$
\boldsymbol{N}_{k}:=\left[\begin{array}{c}
N_{k, 1} \\
N_{k, 2} \\
\vdots \\
N_{k, r}
\end{array}\right], \quad \boldsymbol{N}_{k, \ell}:=\sum_{i=1}^{p} \sum_{j=1}^{X_{r k-\ell, i}}\left(\boldsymbol{\xi}_{r k-\ell+1, j, i}-\mathbb{E}\left(\boldsymbol{\xi}_{r k-\ell+1, j, i}\right)\right), \quad \boldsymbol{\delta}_{k}:=\left[\begin{array}{c}
\epsilon_{r k} \\
\epsilon r k-1 \\
\vdots \\
\epsilon_{r k-r+1}
\end{array}\right] .
$$


Clearly,

$$
\left\|\boldsymbol{M}_{k}\right\|^{2} \leqslant 2\left(\left\|\boldsymbol{N}_{k}\right\|^{2}+\left\|\boldsymbol{\delta}_{k}-\mathbb{E}\left(\boldsymbol{\delta}_{k}\right)\right\|^{2}\right), \quad \mathbb{1}_{\left\{\left\|\boldsymbol{M}_{k}\right\|>n \theta\right\}} \leqslant \mathbb{1}_{\left\{\left\|\boldsymbol{N}_{k}\right\|>n \theta / 2\right\}}+\mathbb{1}_{\left\{\left\|\boldsymbol{\delta}_{k}-\mathbb{E}\left(\boldsymbol{\delta}_{k}\right)\right\|>n \theta / 2\right\}},
$$

hence (5.3) will be proved once we show

$$
\begin{aligned}
& \frac{1}{n^{2}} \sum_{k=1}^{\lfloor n T\rfloor} \mathbb{E}\left(\left\|\boldsymbol{N}_{k}\right\|^{2} \mathbb{1}_{\left\{\left\|\boldsymbol{N}_{k}\right\|>n \theta\right\}} \mid \mathcal{F}_{r k-r}\right) \stackrel{\mathbb{P}}{\longrightarrow} 0 \quad \text { for all } \theta>0, \\
& \frac{1}{n^{2}} \sum_{k=1}^{\lfloor n T\rfloor} \mathbb{E}\left(\left\|\boldsymbol{N}_{k}\right\|^{2} \mathbb{1}_{\left\{\left\|\boldsymbol{\delta}_{k}-\mathbb{E}\left(\boldsymbol{\delta}_{k}\right)\right\|>n \theta\right\}} \mid \mathcal{F}_{r k-r}\right) \stackrel{\mathbb{P}}{\longrightarrow} 0 \quad \text { for all } \theta>0, \\
& \frac{1}{n^{2}} \sum_{k=1}^{\lfloor n T\rfloor} \mathbb{E}\left(\left\|\boldsymbol{\delta}_{k}-\mathbb{E}\left(\boldsymbol{\delta}_{k}\right)\right\|^{2} \mid \mathcal{F}_{r k-r}\right) \stackrel{\mathbb{P}}{\longrightarrow} 0 .
\end{aligned}
$$

First we prove (5.6). Using that the random variables $\left\{\boldsymbol{\xi}_{r k-\ell+1, j, i}: j \in \mathbb{N}, \ell \in\{1, \ldots, r\}, i \in\{1, \ldots, p\}\right\}$ are independent of the $\sigma$-algebra $\mathcal{F}_{r k-r}$ for all $k \in \mathbb{N}$, we get

$$
\mathbb{E}\left(\left\|\boldsymbol{N}_{k}\right\|^{2} \mathbb{1}_{\left\{\left\|\boldsymbol{N}_{k}\right\|>n \theta\right\}} \mid \mathcal{F}_{r k-r}\right)=F_{k}\left(\boldsymbol{X}_{r k-1}, \ldots, \boldsymbol{X}_{r k-r}\right),
$$

where $F_{k}:\left(\mathbb{Z}_{+}^{p}\right)^{r} \rightarrow \mathbb{R}$ is given by

$$
F_{k}\left(\boldsymbol{z}_{1}, \ldots, \boldsymbol{z}_{r}\right):=\mathbb{E}\left(S_{k}\left(\boldsymbol{z}_{1}, \ldots, \boldsymbol{z}_{r}\right)^{2} \mathbb{1}_{\left\{S_{k}\left(\boldsymbol{z}_{1}, \ldots, \boldsymbol{z}_{r}\right)>n \theta\right\}}\right), \quad \boldsymbol{z}_{1}, \ldots, \boldsymbol{z}_{r} \in \mathbb{Z}_{+}^{p},
$$

with

$$
S_{k}\left(\boldsymbol{z}_{1}, \ldots, \boldsymbol{z}_{r}\right):=\left(\sum_{\ell=1}^{r}\left\|\sum_{i=1}^{p} \sum_{j=1}^{z_{\ell, i}}\left(\boldsymbol{\xi}_{r k-\ell+1, j, i}-\mathbb{E}\left(\boldsymbol{\xi}_{r k-\ell+1, j, i}\right)\right)\right\|^{2}\right)^{1 / 2},
$$

where $\boldsymbol{z}_{\ell}=\left(z_{\ell, 1}, \ldots, z_{\ell, p}\right)$. Consider the decomposition

$$
F_{k}\left(\boldsymbol{z}_{1}, \ldots, \boldsymbol{z}_{z}\right)=A_{k}\left(\boldsymbol{z}_{1}, \ldots, \boldsymbol{z}_{r}\right)+B_{k}\left(\boldsymbol{z}_{1}, \ldots, \boldsymbol{z}_{p}\right),
$$

where

$$
\begin{aligned}
& A_{k}\left(\boldsymbol{z}_{1}, \ldots, \boldsymbol{z}_{r}\right):=\sum_{\ell=1}^{r} \sum_{i=1}^{p} \sum_{j=1}^{z_{\ell, i}} \mathbb{E}\left(\left\|\boldsymbol{\xi}_{r k-\ell+1, j, i}-\mathbb{E}\left(\boldsymbol{\xi}_{r k-\ell+1, j, i}\right)\right\|^{2} \mathbb{1}_{\left\{S_{k}\left(\boldsymbol{z}_{1}, \ldots, \boldsymbol{z}_{r}\right)>n \theta\right\}}\right), \\
& B_{k}\left(\boldsymbol{z}_{1}, \ldots, \boldsymbol{z}_{r}\right):=\sum_{\ell=1}^{r} \sum^{\prime} \mathbb{E}\left(\left(\boldsymbol{\xi}_{r k-\ell+1, j, i}-\mathbb{E}\left(\boldsymbol{\xi}_{r k-\ell+1, j, i}\right)\right)^{\top}\left(\boldsymbol{\xi}_{r k-\ell+1, j^{\prime}, i^{\prime}}-\mathbb{E}\left(\boldsymbol{\xi}_{r k-\ell+1, j^{\prime}, i^{\prime}}\right)\right) \mathbb{1}_{\left\{S_{k}\left(\boldsymbol{z}_{1}, \ldots, \boldsymbol{z}_{r}\right)>n \theta\right\}}\right),
\end{aligned}
$$

where the sum $\sum^{\prime}$ is taken for $i, i^{\prime} \in\{1, \ldots, p\}, j \in\left\{1, \ldots, z_{\ell, i}\right\}, j^{\prime} \in\left\{1, \ldots, z_{\ell, i^{\prime}}\right\}$ with $(i, j) \neq\left(i^{\prime}, j^{\prime}\right)$. Consider the inequalities

$$
S_{k}\left(\boldsymbol{z}_{1}, \ldots, \boldsymbol{z}_{r}\right) \leqslant\left\|\boldsymbol{\xi}_{r k-\ell+1, j, i}-\mathbb{E}\left(\boldsymbol{\xi}_{r k-\ell+1, j, i}\right)\right\|+\widetilde{S}_{k, \ell}^{j, i}\left(\boldsymbol{z}_{1}, \ldots, \boldsymbol{z}_{r}\right)
$$

for $\ell \in\{1, \ldots, r\}, i \in\{1, \ldots, p\}$ and $j \in\left\{1, \ldots, z_{\ell^{\prime}, i^{\prime}}\right\}$, where

$$
\widetilde{S}_{k, \ell}^{j, i}\left(\boldsymbol{z}_{1}, \ldots, \boldsymbol{z}_{r}\right):=\sum^{\prime \prime}\left\|\boldsymbol{\xi}_{r k-\ell^{\prime}+1, j^{\prime}, i^{\prime}}-\mathbb{E}\left(\boldsymbol{\xi}_{r k-\ell^{\prime}+1, j^{\prime}, i^{\prime}}\right)\right\|,
$$

where the sum $\sum^{\prime \prime}$ is taken for $\ell^{\prime} \in\{1, \ldots, r\}, i^{\prime} \in\{1, \ldots, p\}$, and $j^{\prime} \in\left\{1, \ldots, z_{\ell^{\prime}, i^{\prime}}\right\}$ with $\left(\ell^{\prime}, j^{\prime}, i^{\prime}\right) \neq(\ell, j, i)$. Using that

$$
\mathbb{1}_{\left\{S_{k}\left(\boldsymbol{z}_{1}, \ldots, \boldsymbol{z}_{r}\right)>n \theta\right\}} \leqslant \mathbb{1}_{\left\{\left\|\boldsymbol{\xi}_{r k-\ell^{\prime}+1, j^{\prime}, i^{\prime}}-\mathbb{E}\left(\boldsymbol{\xi}_{r k-\ell^{\prime}+1, j^{\prime}, i^{\prime}}\right)\right\|>n \theta / 2\right\}}+\mathbb{1}_{\left\{\widetilde{S}_{k, \ell}^{j, i}\left(\boldsymbol{z}_{1}, \ldots, \boldsymbol{z}_{r}\right)>n \theta / 2\right\}},
$$


we have

$$
A_{k}\left(\boldsymbol{z}_{1}, \ldots, \boldsymbol{z}_{r}\right) \leqslant A_{k}^{(1)}\left(\boldsymbol{z}_{1}, \ldots, \boldsymbol{z}_{r}\right)+A_{k}^{(2)}\left(\boldsymbol{z}_{1}, \ldots, \boldsymbol{z}_{r}\right),
$$

where

$$
\begin{aligned}
& A_{k}^{(1)}\left(\boldsymbol{z}_{1}, \ldots, \boldsymbol{z}_{r}\right):=\sum_{\ell=1}^{r} \sum_{i=1}^{p} \sum_{j=1}^{z_{\ell, i}} \mathbb{E}\left(\left\|\boldsymbol{\xi}_{r k-\ell+1, j, i}-\mathbb{E}\left(\boldsymbol{\xi}_{r k-\ell+1, j, i}\right)\right\|^{2} \mathbb{1}_{\left\{\left\|\boldsymbol{\xi}_{r k-\ell+1, j, i}-\mathbb{E}\left(\boldsymbol{\xi}_{r k-\ell+1, j, i}\right)\right\|>n \theta / 2\right\}}\right), \\
& A_{k}^{(2)}\left(\boldsymbol{z}_{1}, \ldots, \boldsymbol{z}_{r}\right):=\sum_{\ell=1}^{r} \sum_{i=1}^{p} \sum_{j=1}^{z_{\ell, i}} \mathbb{E}\left(\left\|\boldsymbol{\xi}_{r k-\ell+1, j, i}-\mathbb{E}\left(\boldsymbol{\xi}_{r k-\ell+1, j, i}\right)\right\|^{2} \mathbb{1}_{\left\{\tilde{S}_{k, \ell}^{j, i}\left(\boldsymbol{z}_{1}, \ldots, \boldsymbol{z}_{r}\right)>n \theta / 2\right\}}\right) .
\end{aligned}
$$

In order to prove (5.6), it is enough to show that

$$
\begin{gathered}
\frac{1}{n^{2}} \sum_{k=1}^{\lfloor n T\rfloor} A_{k}^{(1)}\left(\boldsymbol{X}_{r k-1}, \ldots, \boldsymbol{X}_{r k-r}\right) \stackrel{\mathbb{P}}{\longrightarrow} 0, \quad \frac{1}{n^{2}} \sum_{k=1}^{\lfloor n T\rfloor} A_{k}^{(2)}\left(\boldsymbol{X}_{r k-1}, \ldots, \boldsymbol{X}_{r k-r}\right) \stackrel{\mathbb{P}}{\longrightarrow} 0, \\
\frac{1}{n^{2}} \sum_{k=1}^{\lfloor n T\rfloor} B_{k}\left(\boldsymbol{X}_{r k-r}, \ldots, \boldsymbol{X}_{r k-r}\right) \stackrel{\mathbb{P}}{\longrightarrow} 0
\end{gathered}
$$

as $n \rightarrow \infty$. We have

$$
A_{k}^{(1)}\left(\boldsymbol{z}_{1}, \ldots, \boldsymbol{z}_{r}\right)=\sum_{\ell=1}^{r} \sum_{i=1}^{p} z_{\ell, i} \mathbb{E}\left(\left\|\boldsymbol{\xi}_{r k-\ell+1,1, i}-\mathbb{E}\left(\boldsymbol{\xi}_{r k-\ell+1,1, i}\right)\right\|^{2} \mathbb{1}_{\left\{\left\|\boldsymbol{\xi}_{r k-\ell+1,1, i}-\mathbb{E}\left(\boldsymbol{\xi}_{r k-\ell+1,1, i}\right)\right\|>n \theta / 2\right\}}\right)
$$

for all $k \in \mathbb{N}$, where

$$
\mathbb{E}\left(\left\|\boldsymbol{\xi}_{r k-\ell+1,1, i}-\mathbb{E}\left(\boldsymbol{\xi}_{r k-\ell+1,1, i}\right)\right\|^{2} \mathbb{1}_{\left\{\left\|\boldsymbol{\xi}_{r k-\ell+1,1, i}-\mathbb{E}\left(\boldsymbol{\xi}_{r k-\ell+1,1, i}\right)\right\|>n \theta / 2\right\}}\right) \rightarrow 0 \quad \text { as } n \rightarrow \infty
$$

for all $\ell \in\{1, \ldots, r\}$ and $i \in\{1, \ldots, p\}$ by the dominated convergence theorem. Thus, by Corollary A.2, we get with some constant $K \in \mathbb{R}_{+}$,

$$
\begin{aligned}
\frac{1}{n^{2}} \sum_{k=1}^{\lfloor n T\rfloor} \mathbb{E} & \left(A_{k}^{(1)}\left(\boldsymbol{X}_{r k-1}, \ldots, \boldsymbol{X}_{r k-r}\right)\right) \\
= & \frac{1}{n^{2}} \sum_{k=1}^{\lfloor n T\rfloor} \sum_{\ell=1}^{r} \sum_{i=1}^{p} \sum_{j=1}^{z_{\ell, i}} \mathbb{E}\left(X_{r k-\ell, i}\right) \mathbb{E}\left(\left\|\boldsymbol{\xi}_{1,1, i}-\mathbb{E}\left(\boldsymbol{\xi}_{1,1, i}\right)\right\|^{2} \mathbb{1}_{\left\{\left\|\boldsymbol{\xi}_{1,1, i}-\mathbb{E}\left(\boldsymbol{\xi}_{1,1, i}\right)\right\|>n \theta / 2\right\}}\right) \\
\leqslant & \frac{1}{n^{2}} \sum_{k=1}^{\lfloor n T\rfloor} \sum_{\ell=1}^{r} \sum_{i=1}^{p} K(r k-\ell) \mathbb{E}\left(\left\|\boldsymbol{\xi}_{1,1, i}-\mathbb{E}\left(\boldsymbol{\xi}_{1,1, i}\right)\right\|^{2} \mathbb{1}_{\left\{\left\|\boldsymbol{\xi}_{1,1, i}-\mathbb{E}\left(\boldsymbol{\xi}_{1,1, i}\right)\right\|>n \theta / 2\right\}}\right) \\
= & K \frac{r\lfloor n T\rfloor(r\lfloor n T\rfloor-1)}{2 n^{2}} \sum_{i=1}^{p} \mathbb{E}\left(\left\|\boldsymbol{\xi}_{1,1, i}-\mathbb{E}\left(\boldsymbol{\xi}_{1,1, i}\right)\right\|^{2} \mathbb{1}_{\left\{\left\|\boldsymbol{\xi}_{1,1, i}-\mathbb{E}\left(\boldsymbol{\xi}_{1,1, i}\right)\right\|>n \theta / 2\right\}}\right) \rightarrow 0
\end{aligned}
$$

which yields $n^{-2} \sum_{k=1}^{\lfloor n T\rfloor} A_{k}^{(1)}\left(\boldsymbol{X}_{r k-1}, \ldots, \boldsymbol{X}_{r k-r}\right) \stackrel{\mathbb{P}}{\longrightarrow} 0$.

Independence of $\boldsymbol{\xi}_{r k-\ell+1, j, i}-\mathbb{E}\left(\boldsymbol{\xi}_{r k-\ell+1, j, i}\right)$ and $\widetilde{S}_{k, \ell}^{j, i}\left(\boldsymbol{z}_{1}, \ldots, \boldsymbol{z}_{r}\right)$ implies

$$
A_{k}^{(2)}\left(\boldsymbol{z}_{1}, \ldots, \boldsymbol{z}_{r}\right)=\sum_{\ell=1}^{r} \sum_{i=1}^{p} \sum_{j=1}^{z_{\ell, i}} \mathbb{E}\left(\left\|\boldsymbol{\xi}_{r k-\ell+1, j, i}-\mathbb{E}\left(\boldsymbol{\xi}_{r k-\ell+1, j, i}\right)\right\|^{2}\right) \mathbb{P}\left(\widetilde{S}_{k, \ell}^{j, i}\left(\boldsymbol{z}_{1}, \ldots, \boldsymbol{z}_{r}\right)>n \theta / 2\right) .
$$


Here $\mathbb{E}\left(\left\|\boldsymbol{\xi}_{r k-\ell+1, j, i}-\mathbb{E}\left(\boldsymbol{\xi}_{r k-\ell+1, j, i}\right)\right\|^{2}\right)=\operatorname{tr}\left(\boldsymbol{V}_{\boldsymbol{\xi}_{i}}\right), i \in\{1, \ldots, p\}$, and, by Markov's inequality,

$$
\begin{aligned}
\mathbb{P}\left(\widetilde{S}_{k, \ell}^{j, i}\left(\boldsymbol{z}_{1}, \ldots, \boldsymbol{z}_{r}\right)>n \theta / 2\right) & \leqslant \frac{4}{n^{2} \theta^{2}} \mathbb{E}\left(\widetilde{S}_{k, \ell}^{j, i}\left(\boldsymbol{z}_{1}, \ldots, \boldsymbol{z}_{r}\right)^{2}\right)=\frac{4}{n^{2} \theta^{2}} \operatorname{Var}\left(\widetilde{S}_{k, \ell}^{j, i}\left(\boldsymbol{z}_{1}, \ldots, \boldsymbol{z}_{r}\right)\right) \\
& =\frac{4}{n^{2} \theta^{2}} \sum^{\prime \prime} \operatorname{tr}\left(\boldsymbol{V}_{\boldsymbol{\xi}_{i}}\right) \leqslant \frac{4}{n^{2} \theta^{2}} \sum_{\ell=1}^{r} \sum_{i=1}^{p} z_{\ell, i} \operatorname{tr}\left(\boldsymbol{V}_{\boldsymbol{\xi}_{i}}\right) .
\end{aligned}
$$

Thus we get

$$
A_{k}^{(2)}\left(\boldsymbol{z}_{1}, \ldots, \boldsymbol{z}_{r}\right) \leqslant \frac{4}{n^{2} \theta^{2}} \sum_{\ell=1}^{r} \sum_{i=1}^{p} \sum_{\ell^{\prime}=1}^{r} \sum_{i^{\prime}=1}^{p} z_{\ell, i} z_{\ell^{\prime}, i^{\prime}} \operatorname{tr}\left(\boldsymbol{V}_{\boldsymbol{\xi}_{i}}\right) \operatorname{tr}\left(\boldsymbol{V}_{\boldsymbol{\xi}_{i}^{\prime}}\right) .
$$

Hence, by Cauchy-Schwarz's inequality and Corollary A.2, we get with some constant $K \in \mathbb{R}_{+}$,

$$
\begin{aligned}
\frac{1}{n^{2}} \sum_{k=1}^{\lfloor n T\rfloor} \mathbb{E}\left(A_{k}^{(2)}\left(\boldsymbol{X}_{r k-1}, \ldots, \boldsymbol{X}_{r k-r}\right)\right) & \leqslant \frac{4}{n^{4} \theta^{2}} \sum_{k=1}^{\lfloor n T\rfloor} \sum_{\ell=1}^{r} \sum_{i=1}^{p} \sum_{\ell^{\prime}=1}^{r} \sum_{i^{\prime}=1}^{p} \mathbb{E}\left(\boldsymbol{X}_{r k-\ell, i} \boldsymbol{X}_{r k-\ell^{\prime}, i^{\prime}}\right) \operatorname{tr}\left(\boldsymbol{V}_{\boldsymbol{\xi}_{i}}\right) \operatorname{tr}\left(\boldsymbol{V}_{\boldsymbol{\xi}_{i}^{\prime}}\right) \\
& \leqslant \frac{4 K}{n^{4} \theta^{2}} \sum_{k=1}^{\lfloor n T\rfloor} \sum_{\ell=1}^{r} \sum_{\ell^{\prime}=1}^{r}(r k-\ell)\left(r k-\ell^{\prime}\right)\left(\sum_{i=1}^{p} \operatorname{tr}\left(\boldsymbol{V}_{\boldsymbol{\xi}_{i}}\right)\right)^{2} \rightarrow 0,
\end{aligned}
$$

which implies $n^{-2} \sum_{k=1}^{\lfloor n T\rfloor} A_{k}^{(2)}\left(\boldsymbol{X}_{r k-1}, \ldots, \boldsymbol{X}_{r k-r}\right) \stackrel{\mathbb{P}}{\longrightarrow} 0$. By Cauchy-Schwarz's inequality,

$$
\left|B_{k}\left(\boldsymbol{z}_{1}, \ldots, \boldsymbol{z}_{r}\right)\right| \leqslant \sqrt{B_{k}^{(1)}\left(\boldsymbol{z}_{1}, \ldots, \boldsymbol{z}_{r}\right) \mathbb{E}\left(\mathbb{1}_{\left\{S_{k}\left(\boldsymbol{z}_{1}, \ldots, \boldsymbol{z}_{r}\right)>n \theta\right\}}\right)},
$$

where

$$
B_{k}^{(1)}\left(\boldsymbol{z}_{1}, \ldots, \boldsymbol{z}_{r}\right):=\mathbb{E}\left(\left(\sum_{\ell=1}^{r} \sum^{\prime}\left(\boldsymbol{\xi}_{r k-\ell+1, j, i}-\mathbb{E}\left(\boldsymbol{\xi}_{r k-\ell+1, j, i}\right)\right)^{\top}\left(\boldsymbol{\xi}_{r k-\ell+1, j^{\prime}, i^{\prime}}-\mathbb{E}\left(\boldsymbol{\xi}_{r k-\ell+1, j^{\prime}, i^{\prime}}\right)\right)\right)^{2}\right) .
$$

Using the independence of $\boldsymbol{\xi}_{r k-\ell+1, j, i}-\mathbb{E}\left(\boldsymbol{\xi}_{r k-\ell+1, j, i}\right)$ and $\boldsymbol{\xi}_{r k-\ell+1, j^{\prime}, i^{\prime}}-\mathbb{E}\left(\boldsymbol{\xi}_{r k-\ell+1, j^{\prime}, i^{\prime}}\right)$ for $(i, j) \neq\left(i^{\prime}, j^{\prime}\right)$, we get

$$
\begin{aligned}
B_{k}^{(1)}\left(\boldsymbol{z}_{1}, \ldots, \boldsymbol{z}_{r}\right) & =\sum_{\ell=1}^{r} \sum^{\prime} \operatorname{tr}\left(\boldsymbol{V}_{\boldsymbol{\xi}_{i}}\right) \operatorname{tr}\left(\boldsymbol{V}_{\boldsymbol{\xi}_{i}^{\prime}}\right) \\
& =\sum_{\ell=1}^{r} \sum_{i=1}^{p} z_{\ell, i}\left(z_{\ell, i}-1\right) \operatorname{tr}\left(\boldsymbol{V}_{\boldsymbol{\xi}_{i}}\right)^{2}+\sum_{\ell=1}^{r} \sum_{i \neq i^{\prime}} z_{\ell, i} z_{\ell, i^{\prime}} \operatorname{tr}\left(\boldsymbol{V}_{\boldsymbol{\xi}_{i}}\right) \operatorname{tr}\left(\boldsymbol{V}_{\boldsymbol{\xi}_{i}^{\prime}}\right) \leqslant K_{1}\left(\sum_{\ell=1}^{r} \sum_{i=1}^{p} z_{\ell, i}\right)^{2}
\end{aligned}
$$

with some constant $K_{1} \in \mathbb{R}_{+}$. Further, by Markov's inequality,

$$
\mathbb{E}\left(\mathbb{1}_{\left\{S_{k}\left(\boldsymbol{z}_{1}, \ldots, \boldsymbol{z}_{r}\right)>n \theta\right\}}\right) \leqslant \frac{1}{n^{2} \theta^{2}} \sum_{\ell=1}^{r} \sum_{i=1}^{p} z_{j, i} \operatorname{tr}\left(\boldsymbol{V}_{\boldsymbol{\xi}_{i}}\right) \leqslant \frac{K_{2}}{n^{2} \theta^{2}} \sum_{\ell=1}^{r} \sum_{i=1}^{p} z_{\ell, i}
$$

with some constant $K_{2} \in \mathbb{R}_{+}$. Hence

$$
\left|B_{k}\left(\boldsymbol{z}_{1}, \ldots, \boldsymbol{z}_{r}\right)\right| \leqslant \frac{K_{3}}{n}\left(\sum_{\ell=1}^{r} \sum_{i=1}^{p} z_{\ell, i}\right)^{3 / 2} \leqslant \frac{K_{4}}{n} \sum_{\ell=1}^{r} \sum_{i=1}^{p} z_{\ell, i}^{3 / 2}
$$

with some constants $K_{3}, K_{4} \in \mathbb{R}_{+}$. Thus, in order to show (5.9), it suffices to prove

$$
n^{-3} \sum_{k=1}^{\lfloor n T\rfloor} \sum_{\ell=1}^{r} \sum_{i=1}^{p} X_{r k-\ell, i}^{3 / 2} \stackrel{\mathbb{P}}{\longrightarrow} 0 .
$$


In fact, $n^{-3} \sum_{k=1}^{\lfloor n T\rfloor} \sum_{\ell=1}^{r} \sum_{i=1}^{p} \mathbb{E}\left(X_{r k-\ell, i}^{3 / 2}\right) \rightarrow 0$, since Corollary A.2 implies $\mathbb{E}\left(X_{r k-\ell, i}^{3 / 2}\right) \leqslant\left(\mathbb{E}\left(X_{r k-\ell, i}^{2}\right)\right)^{3 / 4} \leqslant$ $K(r k-\ell)^{3 / 2}$ with some constant $K \in \mathbb{R}_{+}$. Thus we finished the proof of (5.6).

Now we turn to prove (5.7). Using that for all $k \in \mathbb{N}$, the random variables $\left\{\boldsymbol{\xi}_{r k-\ell+1, j, i}, \boldsymbol{\varepsilon}_{r k-\ell+1}: \ell \in\right.$ $\{1, \ldots, r\}, j \in \mathbb{N}, i \in\{1, \ldots, p\}\}$ are independent of the $\sigma$-algebra $\mathcal{F}_{r k-r}$, we get

$$
\mathbb{E}\left(\left\|N_{k}\right\|^{2} \mathbb{1}_{\left\{\left\|\boldsymbol{\delta}_{k}-\mathbb{E}\left(\boldsymbol{\delta}_{k}\right)\right\|>n \theta\right\}} \mid \mathcal{F}_{r k-r}\right)=G_{k}\left(\boldsymbol{X}_{r k-1}, \ldots, \boldsymbol{X}_{r k-r}\right),
$$

where $G_{k}:\left(\mathbb{Z}_{+}^{p}\right)^{r} \rightarrow \mathbb{R}$ is given by

$$
G_{k}\left(\boldsymbol{z}_{1}, \ldots, \boldsymbol{z}_{r}\right):=\mathbb{E}\left(S_{k}\left(\boldsymbol{z}_{1}, \ldots, \boldsymbol{z}_{r}\right)^{2} \mathbb{1}_{\left\{\left\|\boldsymbol{\delta}_{k}-\mathbb{E}\left(\boldsymbol{\delta}_{k}\right)\right\|>n \theta\right\}}\right), \quad \boldsymbol{z}_{1}, \ldots, \boldsymbol{z}_{r} \in \mathbb{Z}_{+}^{p} .
$$

Using again the independence of $\left\{\boldsymbol{\xi}_{r k-\ell+1, j, i}, \boldsymbol{\varepsilon}_{r k-\ell+1}: \ell \in\{1, \ldots, r\}, j \in \mathbb{N}, i \in\{1, \ldots, p\}\right\}$,

$$
G_{k}\left(\boldsymbol{z}_{1}, \ldots, \boldsymbol{z}_{r}\right)=\mathbb{P}\left(\left\|\boldsymbol{\delta}_{k}-\mathbb{E}\left(\boldsymbol{\delta}_{k}\right)\right\|>n \theta\right) \sum_{\ell=1}^{r} \sum_{i=1}^{p} \sum_{j=1}^{z_{\ell, i}} \mathbb{E}\left(\left\|\boldsymbol{\xi}_{r k-\ell+1, j, i}-\mathbb{E}\left(\boldsymbol{\xi}_{r k-\ell+1, j, i}\right)\right\|^{2}\right)
$$

where by Markov's inequality, $\mathbb{P}\left(\left\|\boldsymbol{\delta}_{k}-\mathbb{E}\left(\boldsymbol{\delta}_{k}\right)\right\|>n \theta\right) \leqslant n^{-2} \theta^{-2} \mathbb{E}\left(\left\|\boldsymbol{\delta}_{k}-\mathbb{E}\left(\boldsymbol{\delta}_{k}\right)\right\|^{2}\right)=n^{-2} \theta^{-2} r \operatorname{tr}\left(\boldsymbol{V}_{\boldsymbol{\varepsilon}}\right)$, and $\mathbb{E}\left(\left\|\boldsymbol{\xi}_{r k-\ell+1, j, i}-\mathbb{E}\left(\boldsymbol{\xi}_{r k-\ell+1, j, i}\right)\right\|^{2}\right)=\operatorname{tr}\left(\boldsymbol{V}_{\boldsymbol{\xi}_{i}}\right)$. Hence, in order to show (5.7), it suffices to prove

$$
n^{-4} \sum_{k=1}^{\lfloor n T\rfloor} \sum_{\ell=1}^{r} \sum_{i=1}^{p} X_{r k-\ell, i} \stackrel{\mathbb{P}}{\longrightarrow} 0 .
$$

In fact, by Corollary A.2, $n^{-4} \sum_{k=1}^{\lfloor n T\rfloor} \sum_{\ell=1}^{r} \sum_{i=1}^{p} \mathbb{E}\left(X_{r k-\ell, i}\right) \rightarrow 0$.

Finally we turn to prove (5.8). By independence of $\boldsymbol{\delta}_{k}$ and $\mathcal{F}_{r k-r}$,

$$
\frac{1}{n^{2}} \sum_{k=1}^{\lfloor n T\rfloor} \mathbb{E}\left(\left\|\boldsymbol{\delta}_{k}-\mathbb{E}\left(\boldsymbol{\delta}_{k}\right)\right\|^{2} \mid \mathcal{F}_{r k-r}\right)=\frac{1}{n^{2}} \sum_{k=1}^{\lfloor n T\rfloor} \mathbb{E}\left(\left\|\boldsymbol{\delta}_{k}-\mathbb{E}\left(\boldsymbol{\delta}_{k}\right)\right\|^{2}\right)=\frac{r\lfloor n T\rfloor}{n^{2}} \operatorname{tr}\left(\boldsymbol{V}_{\boldsymbol{\varepsilon}}\right) \rightarrow 0
$$

thus we obtain (5.8). Hence we get (5.3), and we conclude, by Theorem A.3, convergence $\mathcal{M}^{(n)} \stackrel{\mathcal{D}}{\longrightarrow} \mathcal{M}$.

\section{Proof of Theorem 3.6}

In order to prove Theorem 3.6, we want to apply Lemma A.4 using Theorem 3.4. By $(3.10), \boldsymbol{X}^{(n)}=$ $\Psi^{(n)}\left(\mathcal{M}^{(n)}\right)$, where the mapping

$$
\Psi^{(n)}=\left[\begin{array}{c}
\Psi_{1}^{(n)} \\
\vdots \\
\Psi_{r}^{(n)}
\end{array}\right]: \mathrm{D}\left(\mathbb{R}_{+},\left(\mathbb{R}^{p}\right)^{r}\right) \rightarrow \mathrm{D}\left(\mathbb{R}_{+},\left(\mathbb{R}^{p}\right)^{r}\right)
$$

is given by

$$
\begin{aligned}
\Psi_{i}^{(n)}(f)(t):= & \sum_{j=1}^{\lfloor n t\rfloor} \boldsymbol{m}_{\xi}^{(\lfloor n t\rfloor-j) r} \sum_{\ell=i}^{r} \boldsymbol{m}_{\boldsymbol{\xi}}^{\ell-i}\left[f_{\ell}\left(\frac{j}{n}\right)-f_{\ell}\left(\frac{j-1}{n}\right)+\frac{1}{n r} \boldsymbol{m}_{\varepsilon}\right] \\
& +\sum_{j=2}^{\lfloor n t\rfloor} \boldsymbol{m}_{\xi}^{(\lfloor n t\rfloor-j) r} \sum_{\ell=1}^{i-1} \boldsymbol{m}_{\xi}^{\ell-i+r}\left[f_{\ell}\left(\frac{j-1}{n}\right)-f_{\ell}\left(\frac{j-2}{n}\right)+\frac{1}{n r} \boldsymbol{m}_{\varepsilon}\right]
\end{aligned}
$$


for

$$
f=\left[\begin{array}{c}
f_{1} \\
\vdots \\
f_{r}
\end{array}\right] \in \mathrm{D}\left(\mathbb{R}_{+},\left(\mathbb{R}^{p}\right)^{r}\right), \quad t \in \mathbb{R}_{+}, \quad n \in \mathbb{N}, \quad i \in\{1, \ldots, r\} .
$$

Further, $\mathcal{X}=\Psi(\mathcal{M})$, where the mapping $\Psi: \mathrm{D}\left(\mathbb{R}_{+},\left(\mathbb{R}^{p}\right)^{r}\right) \rightarrow \mathrm{D}\left(\mathbb{R}_{+},\left(\mathbb{R}^{p}\right)^{r}\right)$ is given by

$$
\Psi(f)(t):=\left[\begin{array}{c}
\boldsymbol{\Pi} \sum_{\ell=1}^{r} \boldsymbol{m}_{\boldsymbol{\xi}}^{\ell+r-1}\left(f_{\ell}(t)-f_{\ell}(0)+r^{-1} t \boldsymbol{m}_{\boldsymbol{\varepsilon}}\right) \\
\boldsymbol{\Pi} \sum_{\ell=1}^{r} \boldsymbol{m}_{\boldsymbol{\xi}}^{\ell+r-2}\left(f_{\ell}(t)-f_{\ell}(0)+r^{-1} t \boldsymbol{m}_{\boldsymbol{\varepsilon}}\right) \\
\vdots \\
\boldsymbol{\Pi} \sum_{\ell=1}^{r} \boldsymbol{m}_{\boldsymbol{\xi}}^{\ell}\left(f_{\ell}(t)-f_{\ell}(0)+r^{-1} \boldsymbol{t} \boldsymbol{m}_{\boldsymbol{\varepsilon}}\right)
\end{array}\right], \quad f \in \mathrm{D}\left(\mathbb{R}_{+},\left(\mathbb{R}^{p}\right)^{r}\right), \quad t \in \mathbb{R}_{+} .
$$

Measurability of the mappings $\Psi^{(n)}, n \in \mathbb{N}$, and $\Psi$ can be checked as in ([3], p. 603) see Lemma A.5.

The aim of the following discussion is to show that the set $C:=\mathrm{C}\left(\mathbb{R}_{+},\left(\mathbb{R}^{p}\right)^{r}\right)$ satisfies $C \in \mathcal{D}_{\infty}\left(\mathbb{R}_{+},\left(\mathbb{R}^{p}\right)^{r}\right)$, $C \subset C_{\Psi,\left(\Psi^{(n)}\right)_{n \in \mathbb{N}}}$ and $\mathbb{P}(\mathcal{M} \in C)=1$.

First note that $\mathrm{C}\left(\mathbb{R}_{+},\left(\mathbb{R}^{p}\right)^{r}\right) \in \mathcal{D}_{\infty}\left(\mathbb{R}_{+},\left(\mathbb{R}^{p}\right)^{r}\right)$ (see, e.g., Ethier and Kurtz [5], Problem 3.11.25 and Lem. A.6).

Next, we fix a function $f \in C$ and a sequence $\left(f^{(n)}\right)_{n \in \mathbb{N}}$ in $\mathrm{D}\left(\mathbb{R}_{+},\left(\mathbb{R}^{p}\right)^{r}\right)$ with $f^{(n)} \stackrel{\text { lu }}{\longrightarrow} f$. By the definition of $\Psi$, we have $\Psi(f) \in \mathrm{C}\left(\mathbb{R}_{+},\left(\mathbb{R}^{p}\right)^{r}\right)$. Further, we can write

$$
\begin{aligned}
\Psi_{i}^{(n)}\left(f^{(n)}\right)(t)= & \boldsymbol{\Pi} \sum_{\ell=i}^{r} \boldsymbol{m}_{\xi}^{\ell-i}\left[f_{\ell}^{(n)}\left(\frac{\lfloor n t\rfloor}{n}\right)-f_{\ell}^{(n)}(0)+\frac{\lfloor n t\rfloor}{n r} \boldsymbol{m}_{\varepsilon}\right] \\
& +\boldsymbol{\Pi} \sum_{\ell=1}^{i-1} \boldsymbol{m}_{\boldsymbol{\xi}}^{\ell-i+r}\left[f_{\ell}^{(n)}\left(\frac{\lfloor n t\rfloor-1}{n}\right)-f_{\ell}^{(n)}(0)+\frac{\lfloor n t\rfloor-1}{n r} \boldsymbol{m}_{\varepsilon}\right] \\
& +\sum_{j=1}^{\lfloor n t\rfloor}\left(\boldsymbol{m}_{\xi}^{(\lfloor n t\rfloor-j) r}-\boldsymbol{\Pi}\right) \sum_{\ell=i}^{r} \boldsymbol{m}_{\xi}^{\ell-i}\left[f_{\ell}^{(n)}\left(\frac{j}{n}\right)-f_{\ell}^{(n)}\left(\frac{j-1}{n}\right)+\frac{1}{n r} \boldsymbol{m}_{\varepsilon}\right] \\
& +\sum_{j=2}^{\lfloor n t\rfloor}\left(\boldsymbol{m}_{\xi}^{(\lfloor n t\rfloor-j) r}-\boldsymbol{\Pi}\right) \sum_{\ell=1}^{i-1} \boldsymbol{m}_{\boldsymbol{\xi}}^{\ell-i+r}\left[f_{\ell}^{(n)}\left(\frac{j-1}{n}\right)-f_{\ell}^{(n)}\left(\frac{j-2}{n}\right)+\frac{1}{n r} \boldsymbol{m}_{\varepsilon}\right]
\end{aligned}
$$

hence we have

$$
\begin{aligned}
\left\|\Psi_{i}^{(n)}\left(f^{(n)}\right)(t)-\Psi_{i}(f)(t)\right\| \leqslant & K\|\boldsymbol{\Pi}\| \sum_{\ell=i}^{r}\left(\left\|f_{\ell}^{(n)}\left(\frac{\lfloor n t\rfloor}{n}\right)-f_{\ell}(t)\right\|+\left\|f_{\ell}^{(n)}(0)-f_{\ell}(0)\right\|+\frac{1}{n r}\left\|\boldsymbol{m}_{\boldsymbol{\varepsilon}}\right\|\right) \\
& +K\|\boldsymbol{\Pi}\| \sum_{\ell=1}^{i-1}\left(\left\|f_{\ell}^{(n)}\left(\frac{\lfloor n t\rfloor-1}{n}\right)-f_{\ell}(t)\right\|+\left\|f_{\ell}^{(n)}(0)-f_{\ell}(0)\right\|+\frac{2}{n r}\left\|\boldsymbol{m}_{\varepsilon}\right\|\right) \\
& +K \sum_{j=1}^{\lfloor n t\rfloor}\left\|\boldsymbol{m}_{\boldsymbol{\xi}}^{(\lfloor n t\rfloor-j) r}-\boldsymbol{\Pi}\right\| \sum_{\ell=i}^{r}\left(\left\|f_{\ell}^{(n)}\left(\frac{j}{n}\right)-f_{\ell}^{(n)}\left(\frac{j-1}{n}\right)\right\|+\frac{1}{n r}\left\|\boldsymbol{m}_{\varepsilon}\right\|\right) \\
& +K \sum_{j=2}^{\lfloor n t\rfloor}\left\|\boldsymbol{m}_{\xi}^{(\lfloor n t\rfloor-j) r}-\boldsymbol{\Pi}\right\| \sum_{\ell=1}^{i-1}\left(\left\|f_{\ell}^{(n)}\left(\frac{j-1}{n}\right)-f_{\ell}^{(n)}\left(\frac{j-2}{n}\right)\right\|+\frac{1}{n r}\left\|\boldsymbol{m}_{\boldsymbol{\varepsilon}}\right\|\right) .
\end{aligned}
$$

Here for all $T>0$ and $t \in[0, T]$,

$$
\begin{aligned}
\left\|f^{(n)}\left(\frac{\lfloor n t\rfloor}{n}\right)-f(t)\right\| & \leqslant\left\|f^{(n)}\left(\frac{\lfloor n t\rfloor}{n}\right)-f\left(\frac{\lfloor n t\rfloor}{n}\right)\right\|+\left\|f\left(\frac{\lfloor n t\rfloor}{n}\right)-f(t)\right\| \\
& \leqslant \omega_{T}\left(f, n^{-1}\right)+\sup _{t \in[0, T]}\left\|f^{(n)}(t)-f(t)\right\|,
\end{aligned}
$$


where $\omega_{T}(f, \cdot)$ is the modulus of continuity of $f$ on $[0, T]$, and we have $\omega_{T}\left(f, n^{-1}\right) \rightarrow 0$ since $f$ is continuous (see, e.g., Jacod and Shiryaev [10], VI.1.6). In a similar way,

$$
\left\|f^{(n)}\left(\frac{j}{n}\right)-f^{(n)}\left(\frac{j-1}{n}\right)\right\| \leqslant \omega_{T}\left(f, n^{-1}\right)+2 \sup _{t \in[0, T]}\left\|f^{(n)}(t)-f(t)\right\|
$$

for all $j \in\{1, \ldots, n\}$. By $(2.3)$,

$$
\sum_{j=1}^{\lfloor n t\rfloor}\left\|\boldsymbol{m}_{\xi}^{(\lfloor n t\rfloor-j) r}-\boldsymbol{\Pi}\right\| \leqslant \sum_{j=1}^{\lfloor n T\rfloor} c \kappa^{\lfloor n t\rfloor-j} \leqslant \frac{c}{1-\kappa} .
$$

Using that $f^{(n)} \stackrel{\text { lu }}{\longrightarrow} f$ as $n \rightarrow \infty$, we have $\Psi^{(n)}\left(f^{(n)}\right) \stackrel{\text { lu }}{\longrightarrow} \psi(f)$ as $n \rightarrow \infty$. Thus we conclude $C \subset C_{\Psi,\left(\Psi^{(n)}\right)_{n \in \mathbb{N}}}$.

By the definition of a strong solution (see, e.g., [10], Def. 2.24, Chap. III), $\mathcal{M}$ has almost sure continuous sample paths, so we have $\mathbb{P}(\mathcal{M} \in C)=1$. Consequently, by Lemma A.4, we obtain $\boldsymbol{\mathcal { X }}^{(n)}=\Psi^{(n)}\left(\mathcal{M}^{(n)}\right) \stackrel{\mathcal{D}}{\longrightarrow}$ $\Psi(\mathcal{M}) \stackrel{\mathcal{D}}{=} \mathcal{X}$ as $n \rightarrow \infty$.

Clearly, $\boldsymbol{m}_{\xi}^{r} \boldsymbol{\Pi}=\boldsymbol{m}_{\boldsymbol{\xi}}^{r} \lim _{n \rightarrow \infty} \boldsymbol{m}_{\boldsymbol{\xi}}^{n r}=\lim _{n \rightarrow \infty} \boldsymbol{m}_{\boldsymbol{\xi}}^{(n+1) r}=\boldsymbol{\Pi}$, hence $\left(\boldsymbol{m}_{\xi}^{r} \mathcal{Y}_{t}\right)_{t \in \mathbb{R}_{+}}=\left(\mathcal{Y}_{t}\right)_{t \in \mathbb{R}_{+}}$.

\section{Appendix A}

In the proof of Theorem 3.1 we use some facts about the first and second order moments of the sequences $\left(\boldsymbol{X}_{k}\right)_{k \in \mathbb{Z}_{+}}$and $\left(\boldsymbol{M}_{k}\right)_{k \in \mathbb{N}}$.

Lemma A.1. Let $\left(\boldsymbol{X}_{k}\right)_{k \in \mathbb{Z}_{+}}$be a p-type branching process with immigration. Suppose that $\boldsymbol{X}_{0}=\mathbf{0}$, $\mathbb{E}\left(\left\|\boldsymbol{\xi}_{1,1, i}\right\|^{2}\right)<\infty$ for all $i \in\{1, \ldots, p\}$ and $\mathbb{E}\left(\left\|\varepsilon_{1}\right\|^{2}\right)<\infty$. Then

$$
\begin{gathered}
\mathbb{E}\left(\boldsymbol{X}_{k}\right)=\sum_{j=0}^{k-1} \boldsymbol{m}_{\boldsymbol{\xi}}^{j} \boldsymbol{m}_{\boldsymbol{\varepsilon}} \\
\operatorname{Var}\left(\boldsymbol{X}_{k}\right)=\sum_{j=0}^{k-1} \boldsymbol{m}_{\boldsymbol{\xi}}^{j} \boldsymbol{V}_{\boldsymbol{\varepsilon}}\left(\boldsymbol{m}_{\boldsymbol{\xi}}^{\top}\right)^{j}+\sum_{j=0}^{k-2} \boldsymbol{m}_{\boldsymbol{\xi}}^{j} \sum_{\ell=0}^{k-j-2}\left[\left(\boldsymbol{m}_{\boldsymbol{\xi}}^{\ell} \boldsymbol{m}_{\boldsymbol{\varepsilon}}\right) \odot \boldsymbol{V}_{\boldsymbol{\xi}}\right]\left(\boldsymbol{m}_{\boldsymbol{\xi}}^{\top}\right)^{j} .
\end{gathered}
$$

If, in addition, $\left(\boldsymbol{X}_{k}\right)_{k \in \mathbb{Z}_{+}}$is a critical indecomposable -type branching process with immigration and the offspring mean matrix $\boldsymbol{m}_{\boldsymbol{\xi}}$ has the form $(2.1)$, then, for all $k \in \mathbb{N}$, we have $\mathbb{E}\left(\boldsymbol{M}_{k} \mid \mathcal{F}_{r k-r}\right)=\mathbf{0}, \mathbb{E}\left(\boldsymbol{M}_{k}\right)=\mathbf{0}$, and

$$
\begin{aligned}
\mathbb{E}\left(\boldsymbol{M}_{k} \boldsymbol{M}_{k}^{\top} \mid \mathcal{F}_{r k-r}\right) & =\bigoplus_{\ell=1}^{r}\left\{\left[\boldsymbol{m}_{\boldsymbol{\xi}}^{r-\ell} \boldsymbol{X}_{r k-r}+\sum_{j=1}^{r-\ell} \boldsymbol{m}_{\boldsymbol{\xi}}^{j-1} \boldsymbol{m}_{\boldsymbol{\varepsilon}}\right] \odot \boldsymbol{V}_{\boldsymbol{\xi}}+\boldsymbol{V}_{\boldsymbol{\varepsilon}}\right\} \\
\mathbb{E}\left(\boldsymbol{M}_{k} \boldsymbol{M}_{k}^{\top}\right) & =\bigoplus_{\ell=1}^{r}\left\{\left[\boldsymbol{m}_{\boldsymbol{\xi}}^{r-\ell} \mathbb{E}\left(\boldsymbol{X}_{r k-r}\right)+\sum_{j=1}^{r-\ell} \boldsymbol{m}_{\boldsymbol{\xi}}^{j-1} \boldsymbol{m}_{\boldsymbol{\varepsilon}}\right] \odot \boldsymbol{V}_{\boldsymbol{\xi}}+\boldsymbol{V}_{\boldsymbol{\varepsilon}}\right\}
\end{aligned}
$$

Proof. We have already proved (A.1), see (1.4). The equality $\boldsymbol{M}_{k, \ell}=\boldsymbol{X}_{r k-\ell+1}-\mathbb{E}\left(\boldsymbol{X}_{r k-\ell+1} \mid \mathcal{F}_{r k-\ell}\right)$ clearly implies $\mathbb{E}\left(\boldsymbol{M}_{k, \ell} \mid \mathcal{F}_{r k-\ell}\right)=\mathbf{0}$, thus $\mathbb{E}\left(\boldsymbol{M}_{k, \ell} \mid \mathcal{F}_{r k-r}\right)=\mathbb{E}\left[\mathbb{E}\left(\boldsymbol{M}_{k, \ell} \mid \mathcal{F}_{r k-\ell}\right) \mid \mathcal{F}_{r k-r}\right]=\mathbf{0}$, and hence $\mathbb{E}\left(\boldsymbol{M}_{k}\right)=\mathbf{0}$. The proof of (A.2) can be found in Ispány and Pap [8]. By (1.2) and (3.4),

$$
\begin{aligned}
\boldsymbol{M}_{k, \ell} & =\boldsymbol{X}_{r k-\ell+1}-\mathbb{E}\left(\boldsymbol{X}_{r k-\ell+1} \mid \mathcal{F}_{r k-\ell}\right)=\boldsymbol{X}_{r k-\ell+1}-\boldsymbol{m}_{\boldsymbol{\xi}} \boldsymbol{X}_{r k-\ell}-\boldsymbol{m}_{\varepsilon} \\
& =\sum_{i=1}^{p} \sum_{j=1}^{X_{r k-\ell, i}}\left(\boldsymbol{\xi}_{r k-\ell+1, j, i}-\mathbb{E}\left(\boldsymbol{\xi}_{r k-\ell+1, j, i}\right)\right)+\left(\varepsilon_{r k-\ell+1}-\mathbb{E}\left(\boldsymbol{\varepsilon}_{r k-\ell+1}\right)\right) .
\end{aligned}
$$


For each $k \in \mathbb{N}$ and $\ell \in\{1, \ldots, p\}$, the random vectors

$$
\left\{\boldsymbol{\xi}_{r k-\ell+1, j, i}-\mathbb{E}\left(\boldsymbol{\xi}_{r k-\ell+1, j, i}\right), \boldsymbol{\varepsilon}_{r k-\ell+1}-\mathbb{E}\left(\varepsilon_{r k-\ell+1}\right): j \in \mathbb{N}, i \in\{1, \ldots, p\}\right\}
$$

are independent of each others, independent of $\mathcal{F}_{r k-\ell}$, and have zero mean, hence

$$
\mathbb{E}\left(\boldsymbol{M}_{k, \ell} \boldsymbol{M}_{k, \ell}^{\top} \mid \mathcal{F}_{r k-\ell}\right)=\sum_{i=1}^{p} X_{r k-\ell, i} \boldsymbol{V}_{\boldsymbol{\xi}_{i}}+\boldsymbol{V}_{\boldsymbol{\varepsilon}}=\left[\boldsymbol{X}_{r k-\ell} \odot \boldsymbol{V}_{\boldsymbol{\xi}}\right]+\boldsymbol{V}_{\boldsymbol{\varepsilon}} .
$$

By the tower rule and by (1.3),

$$
\mathbb{E}\left(\boldsymbol{M}_{k, \ell} \boldsymbol{M}_{k, \ell}^{\top} \mid \mathcal{F}_{r k-r}\right)=\left[\mathbb{E}\left(\boldsymbol{X}_{r k-\ell} \mid \mathcal{F}_{r k-r}\right) \odot \boldsymbol{V}_{\boldsymbol{\xi}}\right]+\boldsymbol{V}_{\boldsymbol{\varepsilon}}=\left[\boldsymbol{m}_{\boldsymbol{\xi}}^{r-\ell} \boldsymbol{X}_{r k-r}+\sum_{j=1}^{r-\ell} \boldsymbol{m}_{\boldsymbol{\xi}}^{j-1} \boldsymbol{m}_{\boldsymbol{\varepsilon}}\right] \odot \boldsymbol{V}_{\boldsymbol{\xi}}+\boldsymbol{V}_{\boldsymbol{\varepsilon}} .
$$

If $j, \ell \in\{1, \ldots, p\}$ with $j<\ell$ then, again by the tower rule,

$$
\mathbb{E}\left(\boldsymbol{M}_{k, j} \boldsymbol{M}_{k, \ell}^{\top} \mid \mathcal{F}_{r k-r}\right)=\mathbb{E}\left(\boldsymbol{M}_{k, j} \mathbb{E}\left(\boldsymbol{M}_{k, \ell} \mid \mathcal{F}_{r k-\ell}\right)^{\top} \mid \mathcal{F}_{r k-r}\right)=\mathbf{0}
$$

since $\mathbb{E}\left(\boldsymbol{M}_{k, \ell} \mid \mathcal{F}_{r k-\ell}\right)=\mathbf{0}$, and similarly, if $j, \ell \in\{1, \ldots, p\}$ with $j>\ell$ then $\mathbb{E}\left(\boldsymbol{M}_{k, j} \boldsymbol{M}_{k, \ell}^{\top} \mid \mathcal{F}_{r k-r}\right)=\mathbf{0}$, thus we conclude (A.3), and hence, (A.4).

Lemma A.2. Let $\left(\boldsymbol{X}_{k}\right)_{k \in \mathbb{Z}_{+}}$be a critical indecomposable p-type branching process with immigration. Suppose that $\boldsymbol{X}_{0}=\mathbf{0}, \mathbb{E}\left(\left\|\boldsymbol{\xi}_{1,1, i}\right\|^{4}\right)<\infty$ for all $i \in\{1, \ldots, p\}$ and $\mathbb{E}\left(\left\|\varepsilon_{1}\right\|^{4}\right)<\infty$. Then

$$
\mathbb{E}\left(\left\|\boldsymbol{X}_{k}\right\|\right)=\mathrm{O}(k), \quad \mathbb{E}\left(\left\|\boldsymbol{X}_{k}\right\|^{2}\right)=\mathrm{O}\left(k^{2}\right), \quad \mathbb{E}\left(\left\|\boldsymbol{M}_{k}\right\|\right)=\mathrm{O}\left(k^{1 / 2}\right)
$$

Proof. By (A.1),

$$
\left\|\mathbb{E}\left(\boldsymbol{X}_{k}\right)\right\| \leqslant \sum_{j=0}^{k-1}\left\|\boldsymbol{m}_{\boldsymbol{\xi}}^{j}\right\| \cdot\left\|\boldsymbol{m}_{\boldsymbol{\varepsilon}}\right\|=\mathrm{O}(k)
$$

since

$$
C_{\boldsymbol{\xi}}:=\sup _{j \in \mathbb{Z}_{+}}\left\|\boldsymbol{m}_{\xi}^{j}\right\|<\infty .
$$

Indeed, write $j \in \mathbb{Z}_{+}$in the form $j=r k+i$ with $k \in \mathbb{Z}_{+}$and $i \in\{0, \ldots, r-1\}$. Then $\left\|\boldsymbol{m}_{\boldsymbol{\xi}}^{j}\right\|=\left\|\boldsymbol{m}_{\xi}^{r k+i}\right\| \leqslant$ $\left\|\boldsymbol{m}_{\xi}^{r k}\right\|\left\|\boldsymbol{m}_{\boldsymbol{\xi}}^{i}\right\| \leqslant(c+\|\boldsymbol{\Pi}\|) \max _{i \in\{0, \ldots, r-1\}}\left\|\boldsymbol{m}_{\xi}^{i}\right\|=: C_{\boldsymbol{\xi}}<\infty$, since (2.3) implies $\left\|\boldsymbol{m}_{\xi}^{r k}\right\| \leqslant\left\|\boldsymbol{m}_{\boldsymbol{\xi}}^{r k}-\boldsymbol{\Pi}\right\|+\|\boldsymbol{\Pi}\| \leqslant$ $c+\|\boldsymbol{\Pi}\|$.

We have

$$
\mathbb{E}\left(\left\|\boldsymbol{X}_{k}\right\|^{2}\right)=\mathbb{E}\left[\operatorname{tr}\left(\boldsymbol{X}_{k} \boldsymbol{X}_{k}^{\top}\right)\right]=\operatorname{tr}\left(\operatorname{Var}\left(\boldsymbol{X}_{k}\right)\right)+\operatorname{tr}\left[\mathbb{E}\left(\boldsymbol{X}_{k}\right) \mathbb{E}\left(\boldsymbol{X}_{k}\right)^{\top}\right]
$$

where $\operatorname{tr}\left[\mathbb{E}\left(\boldsymbol{X}_{k}\right) \mathbb{E}\left(\boldsymbol{X}_{k}\right)^{\top}\right]=\left\|\mathbb{E}\left(\boldsymbol{X}_{k}\right)\right\|^{2} \leqslant\left[\mathbb{E}\left(\left\|\boldsymbol{X}_{k}\right\|\right)\right]^{2}=\mathrm{O}\left(k^{2}\right)$. Moreover, $\operatorname{tr}\left(\operatorname{Var}\left(\boldsymbol{X}_{k}\right)\right)=\mathrm{O}\left(k^{2}\right)$. Indeed, by (A.2) and (A.6),

$$
\left\|\operatorname{Var}\left(\boldsymbol{X}_{k}\right)\right\| \leqslant\left\|\boldsymbol{V}_{\boldsymbol{\varepsilon}}\right\| \sum_{j=0}^{k-1}\left\|\boldsymbol{m}_{\boldsymbol{\xi}}^{j}\right\|^{2}+\left\|\boldsymbol{m}_{\boldsymbol{\varepsilon}}\right\| \cdot\left\|\boldsymbol{V}_{\boldsymbol{\xi}}\right\| \sum_{j=0}^{k-2}\left\|\boldsymbol{m}_{\boldsymbol{\xi}}^{j}\right\|^{2} \sum_{\ell=0}^{k-j-2}\left\|\boldsymbol{m}_{\boldsymbol{\xi}}^{\ell}\right\| \leqslant C_{\boldsymbol{\xi}}^{2}\left\|\boldsymbol{V}_{\boldsymbol{\varepsilon}}\right\| k+C_{\boldsymbol{\xi}}^{3}\left\|\boldsymbol{m}_{\boldsymbol{\varepsilon}}\right\| \cdot\left\|\boldsymbol{V}_{\boldsymbol{\xi}}\right\| k^{2},
$$

where $\left\|\boldsymbol{V}_{\boldsymbol{\xi}}\right\|:=\sum_{i=1}^{p}\left\|\boldsymbol{V}_{\boldsymbol{\xi}_{i}}\right\|$, hence we obtain $\mathbb{E}\left(\left\|\boldsymbol{X}_{k}\right\|^{2}\right)=O\left(k^{2}\right)$.

We have

$$
\mathbb{E}\left(\left\|\boldsymbol{M}_{k}\right\|\right) \leqslant \sqrt{\mathbb{E}\left(\left\|\boldsymbol{M}_{k}\right\|^{2}\right)}=\sqrt{\mathbb{E}\left[\operatorname{tr}\left(\boldsymbol{M}_{k} \boldsymbol{M}_{k}^{\top}\right)\right]}=\sqrt{\operatorname{tr}\left[\bigoplus_{i=1}^{r}\left\{\left[\boldsymbol{m}_{\boldsymbol{\xi}}^{r-i} \mathbb{E}\left(\boldsymbol{X}_{r k-r}\right)+\sum_{j=1}^{r-i} \boldsymbol{m}_{\boldsymbol{\xi}}^{j-1} \boldsymbol{m}_{\boldsymbol{\varepsilon}}\right] \odot \boldsymbol{V}_{\boldsymbol{\xi}}+\boldsymbol{V}_{\boldsymbol{\varepsilon}}\right\}\right]}
$$

hence we obtain $\mathbb{E}\left(\left\|\boldsymbol{M}_{k}\right\|\right)=\mathrm{O}\left(k^{1 / 2}\right)$ from $\mathbb{E}\left(\left\|\boldsymbol{X}_{k}\right\|\right)=\mathrm{O}(k)$. 
Next we recall a result about convergence of random step processes towards a diffusion process (see [7], Cor. 2.2).

Theorem A.3. Let $\gamma: \mathbb{R}_{+} \times \mathbb{R}^{p} \rightarrow \mathbb{R}^{p \times q}$ be a continuous function. Assume that uniqueness in the sense of probability law holds for the SDE

$$
\mathrm{d} \mathcal{U}_{t}=\gamma\left(t, \mathcal{U}_{t}\right) \mathrm{d} \mathcal{W}_{t}, \quad t \in \mathbb{R}_{+},
$$

with initial value $\mathcal{U}_{0}=\boldsymbol{u}_{0}$ for all $\boldsymbol{u}_{0} \in \mathbb{R}^{p}$, where $\left(\mathcal{W}_{t}\right)_{t \in \mathbb{R}_{+}}$is a q-dimensional standard Wiener process. Let $\left(\mathcal{U}_{t}\right)_{t \in \mathbb{R}_{+}}$be a solution of (A.7) with initial value $\mathcal{U}_{0}=\mathbf{0}$.

For each $n \in \mathbb{N}$, let $\left(\boldsymbol{U}_{k}^{(n)}\right)_{k \in \mathbb{N}}$ be a sequence of $p$-dimensional martingale differences with respect to a filtration $\left(\mathcal{F}_{k}\right)_{k \in \mathbb{Z}_{+}}$. Let

$$
\mathcal{U}_{t}^{(n)}:=\sum_{k=1}^{\lfloor n t\rfloor} \boldsymbol{U}_{k}^{(n)}, \quad t \in \mathbb{R}_{+}, \quad n \in \mathbb{N} .
$$

Suppose $\mathbb{E}\left(\left\|\boldsymbol{U}_{k}^{(n)}\right\|^{2}\right)<\infty$ for all $n, k \in \mathbb{N}$. Suppose that for each $T>0$,

(i) $\sup _{t \in[0, T]}\left\|\sum_{k=1}^{\lfloor n t\rfloor} \mathbb{E}\left[\boldsymbol{U}_{k}^{(n)}\left(\boldsymbol{U}_{k}^{(n)}\right)^{\top} \mid \mathcal{F}_{k-1}\right]-\int_{0}^{t} \gamma\left(s, \mathcal{U}_{s}^{(n)}\right) \boldsymbol{\gamma}\left(s, \mathcal{U}_{s}^{(n)}\right)^{\top} \mathrm{d} s\right\| \stackrel{\mathbb{P}}{\longrightarrow} 0$,

(ii) $\sum_{k=1}^{\lfloor n T\rfloor} \mathbb{E}\left(\left\|\boldsymbol{U}_{k}^{(n)}\right\|^{2} \mathbb{1}_{\left\{\left\|\boldsymbol{U}_{k}^{(n)}\right\|>\theta\right\}} \mid \mathcal{F}_{k-1}\right) \stackrel{\mathbb{P}}{\longrightarrow} 0$ for all $\theta>0$

where $\stackrel{\mathbb{P}}{\longrightarrow}$ denotes convergence in probability. Then $\mathcal{U}^{(n)} \stackrel{\mathcal{D}}{\longrightarrow} \mathcal{U}$ as $n \rightarrow \infty$.

Now we recall a version of the continuous mapping theorem.

For functions $f$ and $f_{n}, n \in \mathbb{N}$, in $\mathrm{D}\left(\mathbb{R}_{+}, \mathbb{R}^{p}\right)$, we write $f_{n} \stackrel{\text { lu }}{\longrightarrow} f$ if $\left(f_{n}\right)_{n \in \mathbb{N}}$ converges to $f$ locally uniformly, i.e., if $\sup _{t \in[0, T]}\left\|f_{n}(t)-f(t)\right\| \rightarrow 0$ as $n \rightarrow \infty$ for all $T>0$. For measurable mappings $\Phi: \mathrm{D}\left(\mathbb{R}_{+}, \mathbb{R}^{p}\right) \rightarrow \mathrm{D}\left(\mathbb{R}_{+}, \mathbb{R}^{q}\right)$ and $\Phi_{n}: \mathrm{D}\left(\mathbb{R}_{+}, \mathbb{R}^{p}\right) \rightarrow \mathrm{D}\left(\mathbb{R}_{+}, \mathbb{R}^{q}\right), n \in \mathbb{N}$, we will denote by $C_{\Phi,\left(\Phi_{n}\right)_{n \in \mathbb{N}}}$ the set of all functions $f \in \mathrm{C}\left(\mathbb{R}_{+}, \mathbb{R}^{p}\right)$ for which $\Phi_{n}\left(f_{n}\right) \rightarrow \Phi(f)$ whenever $f_{n} \stackrel{\text { lu }}{\longrightarrow} f$ with $f_{n} \in \mathrm{D}\left(\mathbb{R}_{+}, \mathbb{R}^{p}\right), n \in \mathbb{N}$.

Lemma A.4. Let $\left(\mathcal{U}_{t}\right)_{t \in \mathbb{R}_{+}}$and $\left(\mathcal{U}_{t}^{(n)}\right)_{t \in \mathbb{R}_{+}}, n \in \mathbb{N}$, be $\mathbb{R}^{p}$-valued stochastic processes with càdlàg paths such that $\mathcal{U}^{(n)} \stackrel{\mathcal{D}}{\longrightarrow} \mathcal{U}$. Let $\Phi: \mathrm{D}\left(\mathbb{R}_{+}, \mathbb{R}^{p}\right) \rightarrow \mathrm{D}\left(\mathbb{R}_{+}, \mathbb{R}^{q}\right)$ and $\Phi_{n}: \mathrm{D}\left(\mathbb{R}_{+}, \mathbb{R}^{p}\right) \rightarrow \mathrm{D}\left(\mathbb{R}_{+}, \mathbb{R}^{q}\right), n \in \mathbb{N}$, be measurable mappings such that there exists $C \subset C_{\Phi,\left(\Phi_{n}\right)_{n \in \mathbb{N}}}$ with $C \in \mathcal{D}_{\infty}\left(\mathbb{R}_{+}, \mathbb{R}^{p}\right)$ and $\mathbb{P}(\mathcal{U} \in C)=1$. Then $\Phi_{n}\left(\mathcal{U}^{(n)}\right) \stackrel{\mathcal{D}}{\longrightarrow} \Phi(\mathcal{U})$.

Lemma A.4 can be considered as a consequence of Theorem 3.27 in Kallenberg [12], and we note that a proof of this lemma can also be found in ([7], Lem. 3.1).

Lemma A.5. The mappings $\Psi^{(n)}, n \in \mathbb{N}$, and $\Psi$ defined in Section 6 are measurable.

Proof. Continuity of $\Psi$ follows from the characterization of convergence in $\mathrm{D}\left(\mathbb{R}_{+},\left(\mathbb{R}^{p}\right)^{r}\right)$ (see, e.g., [5], Prop. 3.5.3) thus we obtain measurability of $\Psi$.

In order to prove measurability of $\Psi^{(n)}$, first we localize it. For each $N \in \mathbb{N}$, consider the stopped mapping $\Psi^{(n, N)}: \mathrm{D}\left(\mathbb{R}_{+},\left(\mathbb{R}^{p}\right)^{r}\right) \rightarrow \mathrm{D}\left(\mathbb{R}_{+},\left(\mathbb{R}^{p}\right)^{r}\right)$ given by $\Psi^{(n, N)}(f)(t):=\Psi^{(n)}(f)(t \wedge N)$ for $f \in \mathrm{D}\left(\mathbb{R}_{+},\left(\mathbb{R}^{p}\right)^{r}\right), t \in \mathbb{R}_{+}$, $n, N \in \mathbb{N}$. Obviously, $\Psi^{(n, N)}(f) \rightarrow \Psi^{(n)}(f)$ in $\mathrm{D}\left(\mathbb{R}_{+},\left(\mathbb{R}^{p}\right)^{r}\right)$ as $N \rightarrow \infty$ for all $f \in \mathrm{D}\left(\mathbb{R}_{+},\left(\mathbb{R}^{p}\right)^{r}\right)$, since for 
all $T>0$ and $N \geqslant T$ we have $\Psi^{(n, N)}(f)(t)=\Psi^{(n)}(f)(t), t \in[0, T]$, and hence $\sup _{t \in[0, T]} \| \Psi^{(n, N)}(f)(t)-$ $\Psi^{(n)}(f)(t) \| \rightarrow 0$ as $N \rightarrow \infty$. Consequently, it suffices to show measurability of $\Psi^{(n, N)}$ for all $n, N \in \mathbb{N}$. We can write $\Psi^{(n, N)}=\Psi^{(n, N, 2)} \circ \Psi^{(n, N, 1)}$, where the mappings $\Psi^{(n, N, 1)}: \mathrm{D}\left(\mathbb{R}_{+},\left(\mathbb{R}^{p}\right)^{r}\right) \rightarrow\left(\left(\mathbb{R}^{p}\right)^{r}\right)^{n N+1}$ and $\Psi^{(n, N, 2)}:\left(\left(\mathbb{R}^{p}\right)^{r}\right)^{n N+1} \rightarrow \mathrm{D}\left(\mathbb{R}_{+},\left(\mathbb{R}^{p}\right)^{r}\right)$ are defined by

$$
\begin{aligned}
\Psi^{(n, N, 1)}(f):= & \left(f(0), f\left(\frac{1}{n}\right), f\left(\frac{2}{n}\right), \ldots, f(N)\right), \\
\Psi^{(n, N, 2)}\left(\boldsymbol{x}_{0}, \boldsymbol{x}_{1}, \ldots, \boldsymbol{x}_{n N}\right)(t):= & \sum_{j=1}^{\lfloor n t\rfloor} \boldsymbol{m}_{\boldsymbol{\xi}}^{(\lfloor n t\rfloor-j) r} \sum_{\ell=i}^{r} \boldsymbol{m}_{\boldsymbol{\xi}}^{\ell-i}\left(\boldsymbol{x}_{j, \ell}-\boldsymbol{x}_{j-1, \ell}+\frac{1}{n r} \boldsymbol{m}_{\boldsymbol{\varepsilon}}\right) \\
& +\sum_{j=2}^{\lfloor n t\rfloor} \boldsymbol{m}_{\boldsymbol{\xi}}^{(\lfloor n t\rfloor-j) r} \sum_{\ell=1}^{i-1} \boldsymbol{m}_{\boldsymbol{\xi}}^{\ell-i+r}\left(\boldsymbol{x}_{j-1, \ell}-\boldsymbol{x}_{j-2, \ell}+\frac{1}{n r} \boldsymbol{m}_{\boldsymbol{\varepsilon}}\right)
\end{aligned}
$$

for $f \in \mathrm{D}\left(\mathbb{R}_{+},\left(\mathbb{R}^{p}\right)^{r}\right), t \in \mathbb{R}_{+}, \boldsymbol{x}=\left(\boldsymbol{x}_{0}, \boldsymbol{x}_{1}, \ldots, \boldsymbol{x}_{n N}\right) \in\left(\left(\mathbb{R}^{p}\right)^{r}\right)^{n N+1}, n, N \in \mathbb{N}$. Measurability of $\Psi^{(n, N, 1)}$ follows from Ethier and Kurtz ([5], Prop. 3.7.1). Next we show continuity of $\Psi_{n}^{(n, N, 2)}$ by checking $\sup _{t \in[0, T]}\left\|\Psi^{(n, N, 2)}\left(\boldsymbol{x}^{(k)}\right)(t)-\Psi^{(n, N, 2)}(\boldsymbol{x})(t)\right\| \rightarrow 0$ as $k \rightarrow \infty$ for all $T>0$ whenever $\boldsymbol{x}^{(k)} \rightarrow \boldsymbol{x}$ in $\left(\left(\mathbb{R}^{p}\right)^{r}\right)^{n N+1}$. This convergence follows from the estimate

$$
\begin{aligned}
\sup _{t \in[0, T]}\left\|\Psi^{(n, N, 2)}\left(\boldsymbol{x}^{(k)}\right)(t)-\Psi_{n}^{(n, N, 2)}(\boldsymbol{x})(t)\right\| \leqslant & C_{\boldsymbol{\xi}}^{2} \sum_{j=1}^{\lfloor n(T \wedge N)\rfloor} \sum_{\ell=i}^{r}\left(\left\|\boldsymbol{x}_{j, \ell}^{(k)}-\boldsymbol{x}_{j, \ell}\right\|+\left\|\boldsymbol{x}_{j-1, \ell}^{(k)}-\boldsymbol{x}_{j-1, \ell}\right\|\right) \\
& +C_{\xi}^{2} \sum_{j=2}^{\lfloor n(T \wedge N)\rfloor} \sum_{\ell=1}^{i-1}\left(\left\|\boldsymbol{x}_{j-1, \ell}^{(k)}-\boldsymbol{x}_{j-1, \ell}\right\|+\left\|\boldsymbol{x}_{j-2, \ell}^{(k)}-\boldsymbol{x}_{j-2, \ell}\right\|\right) .
\end{aligned}
$$

We obtain measurability of both $\Psi^{(n, N, 1)}$ and $\Psi^{(n, N, 2)}$, hence we conclude measurability of $\Psi^{(n, N)}$.

Lemma A.6. The subset $\mathrm{C}\left(\mathbb{R}_{+},\left(\mathbb{R}^{p}\right)^{r}\right) \subset \mathrm{D}\left(\mathbb{R}_{+},\left(\mathbb{R}^{p}\right)^{r}\right)$ is closed, thus measurable, i.e., $\mathrm{C}\left(\mathbb{R}_{+},\left(\mathbb{R}^{p}\right)^{r}\right) \in$ $\mathcal{D}_{\infty}\left(\mathbb{R}_{+},\left(\mathbb{R}^{p}\right)^{r}\right)$.

Proof. The complement $\mathrm{D}\left(\mathbb{R}_{+},\left(\mathbb{R}^{p}\right)^{r}\right) \backslash \mathrm{C}\left(\mathbb{R}_{+},\left(\mathbb{R}^{p}\right)^{r}\right)$ is open. Indeed, each function $f \in \mathrm{D}\left(\mathbb{R}_{+},\left(\mathbb{R}^{p}\right)^{r}\right) \backslash$ $\mathrm{C}\left(\mathbb{R}_{+},\left(\mathbb{R}^{p}\right)^{r}\right)$ is discontinuous at some point $t_{f} \in \mathbb{R}_{+}$, and, by the definition of the metric of $\mathrm{D}\left(\mathbb{R}_{+},\left(\mathbb{R}^{p}\right)^{r}\right)$, there exists $r_{f}>0$ such that all $g \in \mathrm{D}\left(\mathbb{R}_{+},\left(\mathbb{R}^{p}\right)^{r}\right)$ is discontinuous at the point $t_{f} \in \mathbb{R}_{+}$whenever the distance of $g$ from $f$ is less than $r_{f}$. Consequently, the set $\mathrm{D}\left(\mathbb{R}_{+},\left(\mathbb{R}^{p}\right)^{r}\right) \backslash \mathrm{C}\left(\mathbb{R}_{+},\left(\mathbb{R}^{p}\right)^{r}\right)$ is the union of open balls with center $f \in \mathrm{D}\left(\mathbb{R}_{+},\left(\mathbb{R}^{p}\right)^{r}\right) \backslash \mathrm{C}\left(\mathbb{R}_{+},\left(\mathbb{R}^{p}\right)^{r}\right)$ and radius $r_{f}$.

\section{REFERENCES}

[1] K.B. Athreya and P.E. Ney, Branching processes. Springer-Verlag (1972).

[2] R.B. Bapat and T.E.S. Raghavan, Nonnegative matrices and applications. Vol. 64 of Encyclopedia of Mathematics and its Applications. Cambridge University Press (1997).

[3] M. Barczy, M. Ispány and G. Pap, Asymptotic behavior of unstable INAR(p)processes. Stoch. Process. Appl. 121 (2011) 583-608.

[4] R.A. Brualdi and D. Cvetković, A combinatorial approach to matrix theory and its applications. Discrete Mathematics and its Applications. CRC Press, Boca Raton (2009).

[5] S.N. Ethier and T.G. Kurtz, Markov processes. Characterization and convergence. Wiley (1986).

[6] R.A. Horn and Ch.R. Johnson, Matrix Analysis. Cambridge University Press (1985).

[7] M. Ispány and G. Pap, A note on weak convergence of random step processes. Acta Math. Hungar. 126 (2010) $381-395$.

[8] M. Ispány and G. Pap, Asymptotic behavior of critical primitive multi-type branching processes with immigration. Stoch. Anal. Appl. 32 (2014) 727-741.

[9] M. Ispány, K. Körmendi and G. Pap, Asymptotic behavior of CLS estimators for 2-type doubly symmetric critical GaltonWatson processes with immigration. Bernoulli 20 (2014) 2247-2277. 
[10] J. Jacod and A.N. Shiryaev, Limit Theorems for Stochastic Processes, 2nd edition. Springer-Verlag (2003).

[11] A. Joffe and M. Métivier, Weak convergence of sequences of semimartingales with applications to multitype branching processes. Adv. Appl. Probab. 18 (1986) 20-65.

[12] O. Kallenberg, Foundations of Modern Probability. Springer (1997).

[13] I. Karatzas and S.E. Shreve, Brownian Motion and Stochastic Calculus, 2nd ed. Springer (1991).

[14] H. Kesten and B.P. Stigum, Additional limit theorems for indecomposable multidimensional Galton-Watson processes. Ann. Math. Statist. 37 (1966) 1463-1481.

[15] K. Körmendi and G. Pap, Statistical inference of 2-type critical Galton-Watson processes with immigration. Preprint arXiv: $1502.04900(2015)$.

[16] H. Minc, Nonnegative matrices. Wiley-Interscience Series in Discrete Mathematics and Optimization. A Wiley-Interscience Publication, John Wiley \& Sons (1988).

[17] M.P. Quine, The multi-type Galton-Watson process with immigration. J. Appl. Probab. 7 (1970) 411-422.

[18] D. Revuz and M. Yor, Continuous Martingales and Brownian Motion. 3rd edition, corrected 2nd printing. Springer-Verlag (2001).

[19] C.Z. Wei and J. Winnicki, Some asymptotic results for the branching process with immigration. Stoch. Process. Appl. 31 (1989) 261-282. 